\title{
Characteristics of Infection Immunity Regulated by Toxoplasma gondii to Maintain Chronic Infection in the Brain
}

\author{
Young Sang Hwang ${ }^{1 \dagger}$, Ji-Hun Shin ${ }^{1 \dagger}$, Jung-Pyo Yang ${ }^{1}$, Bong-Kwang Jung ${ }^{1,2}$, \\ Sang Hyung Lee ${ }^{3}$ and Eun-Hee Shin ${ }^{1,4 *}$
}

${ }^{1}$ Department of Parasitology and Tropical Medicine, Seoul National University College of Medicine, and Institute of Endemic Diseases, Seoul National University, Seoul, South Korea, ${ }^{2}$ Institute of Parasitic Diseases, Korea Association of Health Promotion, Seoul, South Korea, ${ }^{3}$ Department of Neurosurgery, Seoul National University College of Medicine, SMG-SNU Boramae Medical Center, Seoul, South Korea, ${ }^{4}$ Seoul National University Bundang Hospital, Seongnam, South Korea

OPEN ACCESS

Edited by:

Xun Suo,

China Agricultural

University, China

Reviewed by:

M. Victoria Delpino, National Scientific and Technical Research Council (CONICET),

Argentina

Robin Stephens,

University of Texas Medical Branch, United States

*Correspondence:

Eun-Hee Shin

ehshin@snu.ac.kr

tThese authors have contributed equally to this work.

Specialty section: This article was submitted to Microbial Immunology, a section of the journal

Frontiers in Immunology

Received: 05 November 2017 Accepted: 18 January 2018

Published: 05 February 2018

Citation:

Hwang YS, Shin J-H, Yang J-P, Jung B-K, Lee SH and Shin E-H (2018) Characteristics of Infection Immunity Regulated by Toxoplasma gondii to Maintain Chronic Infection in the Brain.

Front. Immunol. 9:158.

doi: 10.3389/fimmu.2018.00158
To examine the immune environment of chronic Toxoplasma gondii infection in the brain, the characteristics of infection-immunity (premunition) in infection with $T$. gondii strain ME49 were investigated for 12 weeks postinfection (PI). The results showed that neuronal cell death, microglia infiltration and activation, inflammatory and anti-inflammatory cytokine expression, Stat1 phosphorylation, and microglia activation and inflammatory gene transcripts related to M1 polarization in the brain were increased during the acute infection (Al) stage (within 6 weeks Pl), suggesting that innate and cellular inflammatory response activation and neurodegeneration contributed to excessive inflammatory responses. However, these immune responses decreased during the chronic infection (Cl) stage (over 6 weeks Pl) with reductions in phosphorylated STAT1 (pSTAT1) and eosinophilic neurons. Notably, increases were observed in transcripts of T-cell exhaustion markers (TIM3, LAG3, KLRG1, etc.), suppressor of cytokines signaling 1 protein (SOCS1), inhibitory checkpoint molecules (PD-1 and PD-L1), and Arg1 from the Al stage (3 weeks PI), implying active immune intervention under the immune environment of $\mathrm{M} 1$ polarization of microglia and increases in inflammatory cytokine levels. However, when BV-2 microglia were stimulated with $T$. gondii lysate antigens (strain $\mathrm{RH}$ or ME49) in vitro, nitrite production increased and urea production decreased. Furthermore, when BV-2 cells were infected by $T$. gondii tachyzoites (strain $\mathrm{RH}$ or ME49) in vitro, nitric oxide synthase and COX-2 levels decreased, whereas Arg1 levels significantly increased. Moreover, Arg1 expression was higher in ME49 infection than in $\mathrm{RH}$ infection, whereas nitrite production was lower in ME49 infection than in $\mathrm{RH}$ infection. Accordingly, these results strongly suggest that immune triggering of $T$. gondii antigens induces M1 polarization and activation of microglia as well as increase NO production, whereas $T$. gondii infection induces the inhibition of harmful inflammatory responses, even with $\mathrm{M} 1$ polarization and activation of microglia and Th1 inflammatory responses, suggesting a host-parasite relationship through immune regulation during $\mathrm{Cl}$. This is a characteristic of infection immunity in infection with T. gondii in the central nervous system, and SOCS1, a negative regulator of toxoplasmic encephalitis, may play a role in the increase in Arg1 levels to suppress NO production. 


\section{INTRODUCTION}

Toxoplasma gondii is an Apicomplexan pathogen of the central nervous system (CNS) (1). Human T. gondii infection generally occurs via ingestion of oocysts (an environmentally resistant form) released in cat feces or undercooked meat containing tissue cysts (1). Following ingestion, bradyzoites and sporozoites released from cysts and oocysts invade intestinal cells, where they are converted to tachyzoites, which can then be disseminated via the blood or lymphatic system to remote organs and can induce an acute infection (AI) or chronic infection (CI) (1). In general, the brain is the most commonly affected site via congenital transmission and subsequent CI, which elicits lifelong immunity against toxoplasmosis (1). Immune responses to T. gondii infection differ during the proliferative (acute) and dormant (chronic and latent) stages and are dependent on differences in phenotype, virulence, and clinical sequelae of the strains of the clonal lineages, such as the highly virulent strain RH (type I) and the avirulent strain ME49 (type II) (1-3). CI of a type II parasite is maintained by the conversion of tachyzoites into bradyzoites, which produce intracellular tissue cysts. The onset and progression of T. gondii encystation result from both intrinsic preprogramming within the parasite and the immune response of the host, which eventually help to maintain a CI (1).

The AI stage of the RH strain is characterized by marked elevations in serum Th1 cytokine levels, such as interferon (IFN) $-\gamma$, tumor necrosis factor (TNF)- $\alpha$, interleukin (IL)-12, and IL-18, and is followed by a lethal outcome in mice at 8 days postinfection (PI) (2). In contrast, non-lethal infection (ME49 strain) is characterized by modest elevations in Th1 cytokines that lead to control of T. gondii infection and minimal damage to the host (2). More specifically, in the CNS, IFN- $\gamma$ plays a critical role in the prevention of toxoplasmic encephalitis (TE) during the late stage of infection in mice via inhibition of tachyzoite proliferation. However, simultaneous IFN- $\gamma$ activation of microglia may cause tissue injury via the production of toxic metabolites, such as nitric oxide $(\mathrm{NO})(4,5)$. However, neurodegeneration does not commonly occur during CI of $T$. gondii, despite potential NO toxicity (4), possibly to control parasitic proliferation and avoid tissue damage in the infected brain via regulation of the appropriate induction between the cytokines IFN- $\gamma$, IL-10, and transforming growth factor beta (TGF- $\beta$ ), and the toxic mediator $\mathrm{NO}(4,6-8)$.

Microglia, which are a type of glial cell and account for 10-15\% of all cells within the brain parenchyma, are macrophages that reside in the brain and spinal cord, and have plasticity due to

Abbreviations: T. gondii, Toxoplasma gondii; PI, postinfection; CI, chronic infection; (), acute infection; NO, nitric oxide; TGF- $\beta$, transforming growth factor- $\beta$; GM-CSF, granulocyte-macrophage colony-stimulating factor; IL-12, interleukin-12; STAT1, signal transducer and activator of transcription1; SOCS1, suppressor of cytokines signaling 1 protein; MHCII, major histocompatibility complex II; TLA, T. gondii lysate antigen; CNS, central nervous system; TE, toxoplasmic encephalitis; iNOS, nitric oxide synthase; PAMPs, pathogen-associated molecular pattern molecules; DG, hippocampal dentate gyrus; MFI, mean fluorescence intensity; IHC, immunohistochemistry; ME-TLA, lysate antigen of $T$. gondii ME49 strain tachyzoites; (RH-TLA), (COX-2) (Arg1), lysate antigen of T. gondii RH strain tachyzoites. the CNS immune environment and consequently play a key role the regulation of CNS inflammatory reactions, tissue injury, and tissue homeostasis (9). During T. gondii infection, microglia are major effector cells that prevent NO-mediated pathogen proliferation (5). During the immune response of the host, T. gondii infection progresses from an acute stage, where tachyzoites replicates for 2 months PI, to the chronic stage, which is characterized by the formation of dormant cysts after 2 months PI $(4,10,11)$. Simultaneously, life-threatening toxoplasmosis characterized by encephalitis is gradually decreased in the latent stage of T. gondii infection (7). However, the underlying switching mechanisms from the prevention of pathogen proliferation to the inhibition of cellular toxic immune response in the CNS remain unclear, although previous in vitro studies have reported a fractional infection period $(1-8,12-15)$. For example, the cytokines IL-2, IL-12, IFN- $\gamma$, and TNF- $\alpha$, which inhibit the growth of T. gondii, and IL-4, IL-10, and TGF- $\beta$, which are involved in the downregulation of the intracerebral immune response, favor the growth of $T$. gondii and have been implicated in TE (7). Besides, several reports insisted that the decrease in the expression levels of the effector molecules IFN- $\gamma$ and $\mathrm{NO}$ is important for latent infection of $T$. gondii $(4,5,8,15,16)$. Hence, active intervention may be a good strategy for prolonged parasitic survival and establishment of a host-parasite relationship without expulsion of the parasites by the host cells.

Our previous study found that a decrease in NO in Tg2576 mice caused Alzheimer's disease even with inflammation of the brain due to TE (8). In particular, because brain tissue is susceptible to the noxious effects of $\mathrm{NO}$, such as wide-spread neurodegeneration, NO production in T. gondii infection has been widely studied as an important regulator and indicator of both protective effects and tissue damage $(4,5,8,15,16)$. In the CNS, microglial cells lead to broad range of immunoregulatory functions with regulation of NO production to prevent further $T$. gondii proliferation $(1,5,8,15)$. However, despite numerous investigations, the time-specific kinetics of microglia activation in chronically infected normal mice have not been defined. Hence, further studies are needed because T. gondii infection is very chronic in immunocompetent hosts and can recur with time. Unfortunately, no previous study has investigated the immunomodulation in the brain during long-term $\mathrm{CI}$ of $T$. gondii and the key events in the regulation of $\mathrm{CI}$, immunity, and parasite proliferation must be addressed to reduce the harmful effects of CI to brain tissues. As previously revealed, IFN- $\gamma$, TNF- $\alpha$, and IL- 12 were found to inhibit parasitic growth both in vivo and in vitro, and IL-10 and TGF- $\beta$ are important to reduce the excessive inflammatory response of the brain to CI of $T$. gondii $(2-7,12,15)$. The interactions of these cytokines inhibit NO production and promote the conversion of M1-type microglial cells to the M2-type via alternative activation accompanying arginase $1(\operatorname{Arg} 1)$ activity and expression of the mannose receptor type C (CD206) (3, 5, $7,12,15,16)$. The effector molecules of $T$. gondii that induce the polarization of macrophages include Toxoplasma rhoptry kinase ROP16 in the alternative activation pathway and Toxoplasma dense granule protein GRA15 in the classical activation pathway, which activate STAT6 and NF- $\mathrm{kB}$, respectively (3). However, immune modulation may not be limited by the molecular actions 
of T. gondii, but rather may be the result of interactions between the host and parasite to continually change the overall immune response during the latent infection stage due to changes in the immune characteristics and the course of infection. For example, several studies have investigated immune characteristics via genetic manipulation, such as the dense granule protein GRA15KO-T of transgenic T. gondii (Toxoplasma-Cre) in IL-10 $0^{-/-}$mice, but neglected to specify the time-varying infectious immunity during CI in regard to the host-parasite interactions (14, 17-19).

The characteristics of infectious immunity in the T. gondiiinfected brain have been mainly investigated in TE with a focus on the AI stage (20). The most important effector molecules of TE and protection against T. gondii include the IL-12/IFN- $\gamma$ axis, as well as IFN- $\gamma$ through signal transducer and activator of transcription 1 (STAT1) and inducible nitric oxide synthase (iNOS) $(5,12,17)$. Nevertheless, prolonged T. gondii infection results in decreased neurodegeneration and inflammatory immune responses, thus we previously identified components of basic and integrative infection immunity (8). In this respect, we aimed to investigate the processes underlying changes in infection immunity from the AI stage to the CI stage, and identified key responses in the immune regulation between a harmful inflammatory response and restoring neurodegeneration in the brain. To this end, we investigated the changes underlying neurodegeneration during $T$. gondii infection, as well as the activation and polarization of microglial cells as effector cells against T. gondii infection. Also, inflammatory and anti-inflammatory responses, changes in transcript expression patterns during the infection period, induction of $\mathrm{NO}$ and Arg1, an arginine hydrolytic enzyme, and finally in vitro infection of $T$. gondii tachyzoites (RH- or ME49 strain) as well as in vitro stimulation of T. gondii lysate antigen (TLA) (RH- or ME-TLA) were investigated to reveal the differences in immune-triggering events between T. gondii infection in the host-parasite relationship and immune characteristics of TLA as pathogen-associated molecular pattern molecules (PAMPs). The results of the present study are very important and interesting in regard to characterizing the infection immunity processes regulated by the host-parasite relationship through proper control of the protective inflammatory immune response, while reducing the harmful effects of neurodegeneration.

\section{MATERIALS AND METHODS}

\section{Experimental Animals}

Male, 7-week-old, C57BL/6 mice were purchased from the ORIENT BIO Animal Center (Seongnam, South Korea) and housed at room temperature $\left(20-23^{\circ} \mathrm{C}\right)$ on a 12 -h light/dark cycle with ad libitum access to sterilized (irradiated and autoclaved) food and water. All animal experiments were conducted in accordance with the ethical standards of the Institutional Animal Care and Use Committee of Seoul National University (SNU-110315-5).

\section{Ethics Statement}

This study protocol was approved by the Ethics Committee of Seoul National University and conducted in strict accordance with the Guidelines for Animal Experiments (SNUIBC-R110302-1).
All surgeries were performed under anesthesia and all efforts were made to ensure minimal animal suffering.

\section{T. gondii Infection}

C57BL/6 mice (Orient Bio) at the age of 7 weeks were intraperitoneally injected with T. gondii strain ME49 (infection dose of 10 cysts) isolated from T. gondii-infected brains harvested at 3 months PI and housed in the animal facility of Seoul National University College of Medicine. Most experiments were conducted at 3 months PI. Six mice were randomly sacrificed at 0,3 , 6,9 , and 12 weeks PI, and brain tissues were collected for histological examinations, cytokine analysis, and microarray analysis.

\section{Neuronal Degeneration in the Hippocampal Dentate Gyrus (DG) Detected by Hematoxylin and Eosin (H\&E) Staining}

Brain tissues were embedded in paraffin and coronal-sectioned at thickness of $10 \mu \mathrm{m}$ through the hippocampus, mounted, and stained with $H \& E$. Then, the tissue specimens were dehydrated with a graded alcohol series, cleared in xylene, fixed with Canadian balsam (Caedax; Merck, Darmstadt, Germany), and mounted on cover slips. Neuronal degeneration in the hippocampal DG was determined by the detection of eosinophilic neurons under a light microscope (Olympus PM-20; Olympus Corporation, Tokyo, Japan). The number of degenerative cells were counted in photomicrographs obtained with a digital camera (Leica DFC 280, Leica Microsystems, Wetzlar, Germany) attached to a microscope (BX-51; Olympus Corporation) using Image J software ver. $1.46 .^{1}$

\section{Detection of T. gondii Cyst in the Brain by Reverse Transcription Polymerase Chain Reaction (RT-PCR)}

Total RNA was isolated from $T$. gondii-infected mouse brain tissue using the RNeasy Mini kit (Qiagen, Hilden, Germany) according to the manufacturer's protocol. All samples were subjected to RT-PCR using the RT premix reverse transcription kit (Elpis Biotech Inc., Daejeon, South Korea) and MG Taq DNA polymerase (Macrogen, Seoul, South Korea) with the following primer pairs: GAPDH (Primer Bank ID 6679937a1): forward 5'-AGG TCG GTG TGA ACG GAT TTG-3' and reverse 5'-TGT AGA CCA TGT AGT TGA GGT CA-3' (123 bp); T. gondii B1 gene: forward 5'-TCC CCT CTG CTG GCG AAA AGT-3' and reverse 5'-AGC GTT CGT GGT CAA CTA TCG ATT G-3' (98 bp). RT-PCR was performed for 35 cycles with an annealing temperature of $54-55^{\circ} \mathrm{C}$ and the products were analyzed by $1 \%$ agarose gel electrophoresis.

\section{Real-time PCR}

Total RNA was isolated from Total RNA was isolated from T. gondii-infected mouse brain tissue using the RNeasy Mini kit (Qiagen, Hilden, Germany) according to the manufacturer's protocol. All samples were reverse transcribed using RT premix

${ }^{1}$ http://pga.mgh.harvard.edu/primerbank/. 
(Elpis Biotech Inc.). Real-time PCR was performed using the CFX96 (Bio-Rad) and SYBR green (Enzynomics ${ }^{\mathrm{TM}}$, Daejeon, Korea) was used to detect amplification products. The reaction conditions used were; initial denaturation at $95^{\circ} \mathrm{C}$ for $15 \mathrm{~min}, 40$ amplification cycles [denaturation at $95^{\circ} \mathrm{C}$ for $10 \mathrm{~s}$, annealing at $60^{\circ} \mathrm{C}$ for $20 \mathrm{~s}$, and extension at $72^{\circ} \mathrm{C}$ for $30 \mathrm{~s}$, followed by melting curve analysis. Data analysis was performed using CFX96 software (Bio-Rad). The following primer sequences were used for real-time PCR: GAPDH: forward 5'-GGT GAA GGT CGG TGT GAA CG-3' and reverse 5'-CTC GCT CCT GGA AGA TGG TG-3' (578 bp); and Socs1: forward 5'-CTG CGG CTT CTA TTG GGG AC-3' and reverse 5'-AAA AGG CAG TCG AAG GTC TCG-3' (217 bp); and Nos2 (iNOS): forward 5' -CAG CAC AGA ATG TTC CAG AAT CC-3' and reverse 5' ${ }^{\prime}$ TGT CAT GCA AAA TCT CTC CAC TGC-3' (105 bp); and Arg1: forward 5'-CTT TAA CCT TGG CTT GCT TCG GAA-3' and reverse 5'-CTT AGT TCT GTC TGC TTT GCT GTG-3' (140 bp).

\section{Immunostaining of T. gondii-Infected Mouse Brain Tissues}

Mice were sacrificed by $\mathrm{CO}_{2}$ asphyxiation at the pre-determined times and brain tissues were collected, then fixed in formalin and embedded in paraffin using the Leica TP1020 Tissue Processor (Leica Microsystems GmbH, Wetzlar, Germany). For specific immunostaining, samples were immunostained using the ChromoMap Kit and the Discovery XT automated staining instrument (Ventana Medical Systems, Inc., Tucson, AZ, USA). Briefly, $4 \mu \mathrm{m}$-thick tissue sections were fixed on Probe-OnPlus Slides (Thermo Fisher Scientific, Swedesboro, NJ, USA), deparaffinized in xylene, rehydrated in a graded series of alcohol $(100,95,80$, and 70$)$, and finally rinsed with distilled water. After antigen retrieval, the endogenous peroxidase activity of the samples was blocked by treatment of $\mathrm{H}_{2} \mathrm{O}_{2}$ with blocking buffer [1\% fetal bovine serum in phosphate-buffered saline (PBS)] for $30 \mathrm{~min}$. Then, the samples were incubated for $60 \mathrm{~min}$ at room temperature with either rabbit anti-mouse Iba-1 (1:2000, Wako Chemicals, Richmond, VA, USA) or anti-phospho-STAT1 (Tyr701) (58D6, rabbit mAb) (1:500, Cell Signaling Technology, Beverly, MA, USA) primary antibodies, washed thrice with Tris-buffered saline, and incubated with secondary antibodies in UltraMap anti-Rb horseradish peroxidase (HRP) (Ventana Medical Systems, Inc.). Streptavidin-biotin-HRP complex (sABC/HRP) was detected with the ChromoMap DAB detection kit (Ventana Medical System, Inc.). The immunostained sections were then counterstained with hematoxylin (Ventana Medical System, Inc.) and plated on cover slips using Canadian balsam solution (Polysciences Inc., Warrington, PA, USA). Reactions were observed using a light microscope (PM-20; Olympus Corporation) and photomicrographs were acquired with a BX-51 microscope (Olympus Corporation) equipped with a color digital camera (DFC280; Leica Microsystems).

\section{Levels of Inflammatory and Anti-inflammatory Cytokines in T. gondii-Infected Brain Tissue}

Cytokine levels of IL-6, IL-12 (p70), IFN- $\gamma$, TNF- $\alpha$, granulocytemacrophage colony-stimulating factor (GM-CSF), IL-10, IL-4, and
TGF- $\beta$ in T. gondii-infected mouse brains were examined using the Bio-Plex mouse cytokine assay kit (Bio-Rad Laboratories, Hercules, CA, USA) and enzyme-linked immunosorbent assay (ELISA) kits (R\&D Systems, Inc., Minneapolis, MN, USA). Brain tissues of C57BL/ 6 mice were obtained at $0,3,6,9$, and 12 weeks PI, and lysed using the MicroRotofor Cell Lysis Kit (Bio-Rad Laboratories) $(n=3)$. The soluble homogenate proteins were quantified using a bicinchoninic acid (BCA) assay kit (Pierce Biotechnology, Inc., Rockford, IL, USA). The Bio-Plex assay was performed according to the manufacturer's instructions and the raw data [mean fluorescent intensities (MFI)] were analyzed with Bio-Plex Manager Software (Bio-Rad Laboratories) to obtain concentration values. The one-way analysis of variance (ANOVA) was used for statistical analysis, and the results were analyzed using Bio-Plex Data Pro ${ }^{\mathrm{TM}}$ software. Cytokine analysis was performed using ELISA kits (R\&D Systems, Inc.) according to the manufacturer's protocol. The reaction was measured at $450 \mathrm{~nm}$ using an Epoch microplate reader (BioTek Instruments, Inc., Winooski, VT, USA) and cytokine concentrations were calculated using a standard curve of the corresponding cytokine provided with the ELISA kit.

\section{Microarray Analysis of T. gondii-Infected Brain}

Total RNA of $T$. gondii-infected brain tissues was separately extracted at $0,3,6,9$, and 12 weeks PI, and pooled for microarray analysis $(n=3)$, which was performed by Macrogen Inc. (Seoul, South Korea) using an Illumina MouseRef-8 v2 Expression BeadChip array (Illumina, Inc., San Diego, CA, USA). Briefly, $0.55 \mu \mathrm{g}$ of total RNA was amplified using the Illumina TotalPrep RNA Amplification Kit (Ambion, Austin, TX, USA) and purified using the Ambion Illumina RNA amplification kit (Ambion) to yield biotinylated cRNA. Following fragmentation, $0.75 \mu \mathrm{g}$ of cRNA were hybridized to the Mouse Expression BeadChip (Illumina, Inc.) according to the manufacturer's protocol. Arrays were scanned with the Illumina Bead Array Reader Confocal Scanner. Array data export processing and analysis was performed using Illumina GenomeStudio v2011.1 (Gene Expression Module v1.9.0), and the data were analyzed with $\mathrm{R}$ v. 2.15.1 statistical software. Hierarchical cluster analysis was performed using Permute Matrix EN software. All heat maps were generated using Excel Spreadsheet Software (Microsoft Corporation, Redmond, WA, USA) with conditional formatting. The expression rates of each gene at 3, 6, 9 and 12 weeks PI were compared to baseline (week 0 PI). Positive correlations are depicted in yellow (increased expression) and negative correlations (decreased expression) are depicted in blue. Heat maps of inflammatory and anti-inflammatory cytokines, microglia phenotype markers, and immune regulatory markers are represented by color scales. Each row represents cytokines and immune markers, and each column represents infection times from 0 to 12 weeks PI. The color scale of the heat map corresponds the relative minimum (-3) and maximum $(+3)$ values of each cytokine.

\section{TLAs Prepared from Tachyzoites of T. gondii Strains RH and ME49}

Toxoplasma gondii lysate antigens of strain RH or ME49 were prepared as previously described with slight modifications $(8,11)$. 
Briefly, peritoneal exudates of infected mice at day 4 PI were passed twice through a 25-gauge needle and then through a 5- $\mu \mathrm{m}$ Millex filter membrane (Merck Millipore, Tullagreen, Ireland) to remove debris and host cells. Then, tachyzoites of T. gondii strain $\mathrm{RH}$ were recovered and washed with sterilized PBS ( $\mathrm{pH} 7.2)$. Parasites were then washed and suspended in PBS ( $\mathrm{pH}$ 7.2) for further antigen preparation. To prepare ME49 tachyzoite antigens, 20 cysts of strain ME49 were intraperitoneally injected to $\mathrm{BALB} / \mathrm{c}$ mice to obtain ME49 tachyzoites converted from cysts. At 6 to 8 days PI, the tachyzoites were harvested by washing the peritoneal cavity with PBS. Tachyzoites of both T. gondii strains RH and ME49 were cultured in Vero cells (monkey kidney cells, KCLB cell line; no. 10081) grown in complete Roswell Park Memorial Institute 1640 media (WelGENE Inc., Daegu, Korea) supplemented with $100 \mu \mathrm{g} / \mathrm{mL}$ of penicillin (Gibco/BRL, Grand Island, NY, USA), $100 \mu \mathrm{g} / \mathrm{mL}$ of streptomycin (Gibco/BRL), and $5 \%$ fetal calf serum (Lonza, Walkersville, $\mathrm{MD}, \mathrm{USA}$ ) at $37^{\circ} \mathrm{C}$ under an atmosphere of $5 \% \mathrm{CO}_{2}$. After culturing in Vero cells, tachyzoites of strains ME49 and $\mathrm{RH}$ were passed through a 25-gauge needle twice, and then debris and cells were removed by passing through $5-\mu \mathrm{m}$ filter membranes. After washing, the tachyzoites were re-suspended in PBS, sonicated on ice, and then centrifuged. Supernatants, containing the TLA fractions (respectively named as RH-TLA and ME-TLA) were filtered through $0.22-\mu \mathrm{m}$ filter membranes (Millipore Corp., Bedford, MA, USA). Proteins in the TLA fraction were quantified using a BCA commercial reagent (Pierce Biotechnology, Inc.) and stored at $-80^{\circ} \mathrm{C}$ until used.

\section{Flow Cytometry Analysis to Determine the Microglia Phenotype of BV-2 Cells after Treatment of the $T$. gondii Antigen}

Antigens against T. gondii strain $\mathrm{RH}$ or ME49 tachyzoites $(40 \mu \mathrm{g} / \mathrm{mL})$ and/or recombinant IFN- $\gamma(100 \mathrm{ng} / \mathrm{mL}$; PeproTech, Rocky Hill, NJ, USA) were used for in vitro activation of BV-2 cells, a murine microglial cell line. BV-2 cells were cultured in Dulbecco's modified essential medium (Applied Scientific, San Francisco, CA, USA) supplemented with $10 \%$ heat-inactivated fetal calf serum (Hyclone, Ogden, UT, USA), $4 \mathrm{mM}$ L-glutamine, $0.2 \mathrm{mM}$ penicillin, $0.05 \mathrm{mM}$ streptomycin, and $20 \mathrm{mM}$ HEPES (Sigma-Aldrich Corporation, St. Louis, MO, USA) at $37^{\circ} \mathrm{C}$ under an atmosphere of $5 \% \mathrm{CO}_{2}$ (8). After incubation for $24 \mathrm{~h}, \mathrm{BV}-2$ cells were harvested for further fluorescence-activated cell sorting (FACS) analysis. The following anti-mouse antibodies were used for flow cytometry analysis of the cultured BV-2 microglial cells: fluorescein isothiocyanate (FITC)-conjugated anti-CD80 (eBioscience, Inc., San Diego, CA, USA), phycoerythrin (PE)conjugated anti-CD86 (eBioscience, Inc.), FITC-conjugated anti-CD274 (PD-L1) (B7-H1; eBioscience, Inc.), PE-conjugated anti-CD273 (PD-L2) (B7-DC; eBioscience, Inc.), allophycocyanin (APC)-conjugated anti-major histocompatibility complex (MHC) II (CD74, eBioscience), PE-conjugated anti-CD40 (eBioscience, Inc.), and FITC-conjugated anti-CD206 (BioLegend, San Diego, CA, USA). All staining processes for FACS analysis were conducted in accordance with the manufacturer's protocols with FACS staining buffer (PBS containing 1\% bovine serum albumin and $0.1 \%$ sodium azide). Samples were analyzed using a FACSCalibur flow cytometer (BD Immunocytometry Systems, San Jose, CA, USA) with forward/side scatter gates to exclude nonviable cells and the data were analyzed using FlowJo software (Tree Star, Inc., Ashland, OR, USA). Data are presented as the mean $( \pm \mathrm{SD})$ fluorescence intensity (MFI). In this study, in vitro experiment of BV-2 cell culture was data obtained after conducting three individual experiments.

\section{Western Blot Analysis}

Total proteins were extracted from uninfected and infected whole mouse brains using the PRO-PREP ${ }^{\mathrm{TM}}$ Protein Extraction Kit (iNtRON Biotechnology, Seongnam-Si, Korea) and quantified with a NanoDrop spectrophotometer (NanoDrop Technologies, Oxfordshire, UK). Proteins were separated by sodium dodecyl sulfate-10\% polyacrylamide gel electrophoresis at $100 \mathrm{~V}$ for $110 \mathrm{~min}$ and then transferred to a nitrocellulose membrane (BioRad Laboratories) using the Mini Trans-Blot ${ }^{\circledR}$ Electrophoretic Transfer Cell (Bio-Rad Laboratories) at $80 \mathrm{~V}$ for $100 \mathrm{~min}$. The membranes were then incubated with the primary antibodies goat anti-SOCS1 (ab9870; Abcam, Cambridge, UK) (1:200) and mouse anti- $\beta$-actin (sc-47778; dilution, 1:500; Santa Cruz Biotechnology, Dallas, TX, USA), followed by the secondary antibodies donkey anti-goat immunoglobulin (Ig)G-HRP (sc-2020; dilution, 1:2,000; Santa Cruz Biotechnology) and goat anti-mouse IgG-HRP (sc2005; dilution, 1:4,000; Santa Cruz Biotechnology). Signals were detected by exposing the membrane to chemiluminescence HRP substrate (Thermo Fisher Scientific) using a Fuji LAS1000 Lumino Image Analyzer (Fujifilm Corporation, Tokyo, Japan).

\section{Nitrite and Urea Production by BV-2 Cells Stimulated by Various Cytokines and Strain-Specific Tachyzoite Antigens (RH-TLA and ME-TLA)}

BV-2 cells were incubated for $24 \mathrm{~h}$ in 6-well culture plates (SPL Lifesciences Co., Ltd., Pocheon, South Korea) with either $100 \mathrm{ng} /$ $\mathrm{mL}$ of IFN- $\gamma$ (PeproTech), $20 \mathrm{ng} / \mathrm{mL}$ of IL-4 (Prospec-Tany Technogene Ltd., Rehovot, Israel), and/or one of the T. gondii antigens (RH-TLA or ME-TLA) at a concentration of 20 or $40 \mu \mathrm{g} /$ $\mathrm{mL}$. Culture supernatants were collected and assayed to determine contents of nitrite and urea, which reflect NO production and Arg1 levels, respectively. NO production was determined using Griess reagent (Sigma-Aldrich Corporation). After $50 \mu \mathrm{L}$ of culture supernatant was reacted with $50 \mu \mathrm{L}$ of Griess reagent in each well of a 96-well plate (SPL Lifesciences Co., Ltd.), the reaction was measured using a microplate reader at an optical density (OD) of $540 \mathrm{~nm}$ (Biotech, VT, USA). Urea concentration was determined using a commercial urea kit (Abnova Corporation, Taipei, Taiwan) according to the manufacturer's protocol. In brief, $50 \mu \mathrm{L}$ of culture supernatant was mixed with $50 \mu \mathrm{L}$ of distilled water and the solution was reacted with $200 \mu \mathrm{L}$ of a working reagent for $20 \mathrm{~min}$. Then, the $\mathrm{OD}$ value of the sample was measured at $520 \mathrm{~nm}$ and the concentrations of nitrite and urea were calculated using standard curves. In vitro experiment of BV-2 cell culture was data obtained after conducting three individual experiments. 


\section{mRNA Levels of iNOS, COX-2, and Arg1 in T. gondii-Infected BV-2 Cells}

BV-2 cells were infected with tachyzoites of $T$. gondii strain RH or ME49 in vitro at an effector to target ratio (E:T ratio; T. gondii:BV-2 cell) of 1:5 for $24 \mathrm{~h}$. Total RNA of the cultured cells was extracted using the RNeasy kit (Qiagen). All samples were reverse transcribed using RT premix (Elpis Biotech Inc.) and the iQ5 real-time PCR detection system (Bio-Rad Laboratories) with SYBR green master mix (Enzynomics, Cheongju, South Korea) under the following condition: initial denaturation at $50^{\circ} \mathrm{C}$ for $5 \mathrm{~min}$ and $95^{\circ} \mathrm{C}$ for $10 \mathrm{~min}$, followed by 40 amplification cycles of denaturation at $95^{\circ} \mathrm{C}$ for $10 \mathrm{~s}$ and annealing at $60^{\circ} \mathrm{C}$ for $30 \mathrm{~s}$, with a final extension cycle at $72^{\circ} \mathrm{C}$ for $5 \mathrm{~min}$. Specific amplification was verified by analysis of the melting curve and separation of the RT-PCR products on a $3 \%$ agarose gel. Data analysis was performed using $\mathrm{iQ}^{\mathrm{TM}} 5$ optical system software (Bio-Rad Laboratories). The following primer sequences and amplicon sizes were retrieved from the PrimerBank database 2 : GAPDH (PrimerBank ID 6679937a1) forward 5' -AGG TCG GTG TGA ACG GAT TTG-3' and reverse 5'-TGT AGA CCA TGT AGT TGA GGT CA-3' (123 bp); Arg1 (PrimerBank ID7106255a1) forward 5'-CTC CAA GCC AAA GTC CTT AGA G- $3^{\prime}$ and reverse $5^{\prime}$-AGG AGC TGT CAT TAG GGA CAT C-3' (185 bp); Nos2 (iNOS) forward 5'-GTT CTC AGC CCA ACA ATA CAA GA-3'and reverse 5'-GTG GAC GGG TCG ATG TCA C-3'(127 bp); and Ptgs2 (COX-2) forward 5'-TGT GAC TGT ACC CGG ACT GG-3' and reverse $5^{\prime}$-TGC ACA TTG TAA GTA GGT GGA C-3'(233 bp).

\section{Statistical Analysis}

All statistical analyses were performed using Microsoft Excel and GraphPad Prism 5 software (GraphPad Software, Inc., La Jolla, CA, USA). Data are presented as the mean $\pm \mathrm{SD}$. One-way ANOVA followed by the Bonferroni multiple-comparison test were used to assess differences between experimental groups. A probability $(p)$ values of $<0.05$ was considered statistically significant. ${ }^{*}$ indicates significant difference by one-way ANOVA compared with the control. ${ }^{*}$ indicates significant difference between the experimental groups.

\section{RESULTS}

\section{Histopathological Changes and Microglia Activation in the Hippocampal DG during the Al to $\mathrm{Cl}$ Stage of T. gondii}

To observe the neuronal cell damage caused by T. gondii infection, histopathologic changes in the hippocampal region were examined by H\&E staining. As shown in Figure 1A, purplecolored normal and un-injured neurons were observed with a light microscopic throughout most of the hippocampal DG (Figure 1A, week 0), whereas T. gondii infection resulted in an increase the proportion of eosinophilic neurons, which were characterized by cell body shrinkage, intensely stained eosinophilic cytoplasm, and small/shrunken darkly stained nuclei

${ }^{2}$ http://pga.mgh.harvard.edu/primerbank/.

\section{A H\&E-stain}
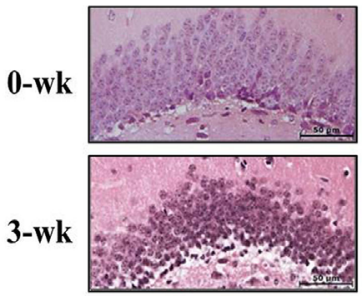

6-wk

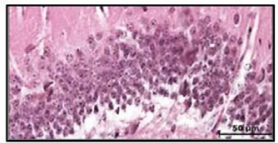

9-wk

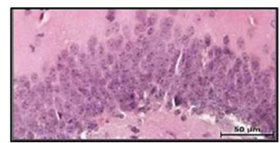

12-wk

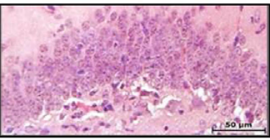

B IHC (Iba-1-Ab)
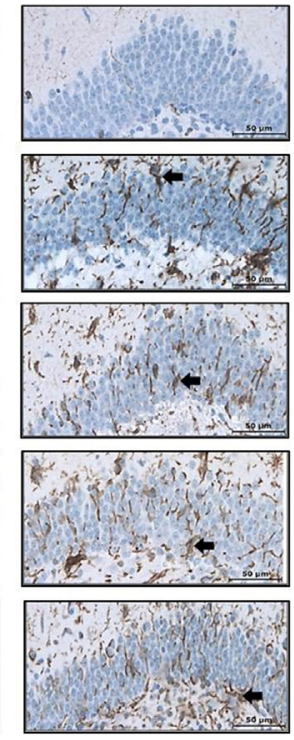

\section{Degenerated cells $(\%)$}

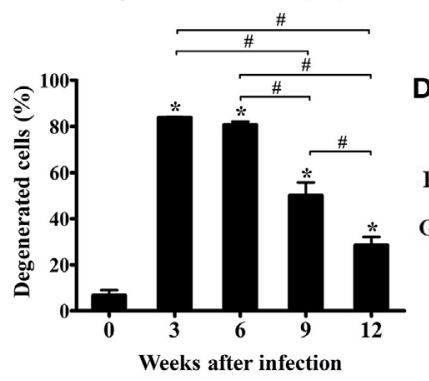

D T. gondii infection in the brain

FIGURE 1 | Changes in neuronal degeneration and proliferation of microglial cells in the hippocampal dentate gyrus during $T$. gondii infection. The brain tissues were harvested at 0, 3, 6, 9, and 12 weeks postinfection, and subjected to histological staining. (A) hematoxylin and eosin staining, 400x. (B) Immunohistochemistry $(\mathrm{IHC})$ results of the brain tissue stained with Iba-1-antibody (brown color), 400x. Scale bar $=50 \mu \mathrm{m}$. (C) Degenerated cells in the brain tissues after $T$. gondii infection were counted and are expressed by percentage (\%). ${ }^{*}$ indicates significant difference compared with the control. " indicates significant difference between the experimental groups. (D) T. gondii-specific B1 gene expression and RT-PCR products were loaded into $1 \%$ agarose gels (98 bp).

(Figure 1A, at 3 and 6 weeks PI). The appearance of eosinophilic neurons was increased during the AI stage and then significantly decreased during the CI stage from $6.7 \pm 3.88 \%$ at week 0 to $83.8 \pm 0.57 \%$ at week $3,80.8 \pm 2.38 \%$ at week $6,50.1 \pm 9.66$ at week 9 , and $28.5 \pm 6.13 \%$ at week 12 PI (Figure 1C, $p<0.05$ ). At this time, activation and infiltration of microglial cells in the DG were evaluated by immunohistochemical analysis with antibody against Iba-1, an activation marker of microglia (Figure 1B). The infiltration of Iba-1-positive cells (colored in brown) during hippocampal formation had increased from 3 weeks PI and was sustained during the 12-week experimental period (Figure 1B). These histopathological changes appeared after infection of $T$. gondii, which were detected by monitoring of the B1 gene (Figure 1D). Importantly, this result suggests that 
neurodegeneration increases during the AI stage and decreases in the CI stage ( 9 weeks PI), despite the presence of harmful signs in the brain tissue, such as activation of inflammatory microglia and continuity of T. gondii infection. Accordingly, further analysis showed that neurodegeneration accompanying T. gondii infection had decreased during the CI stage.
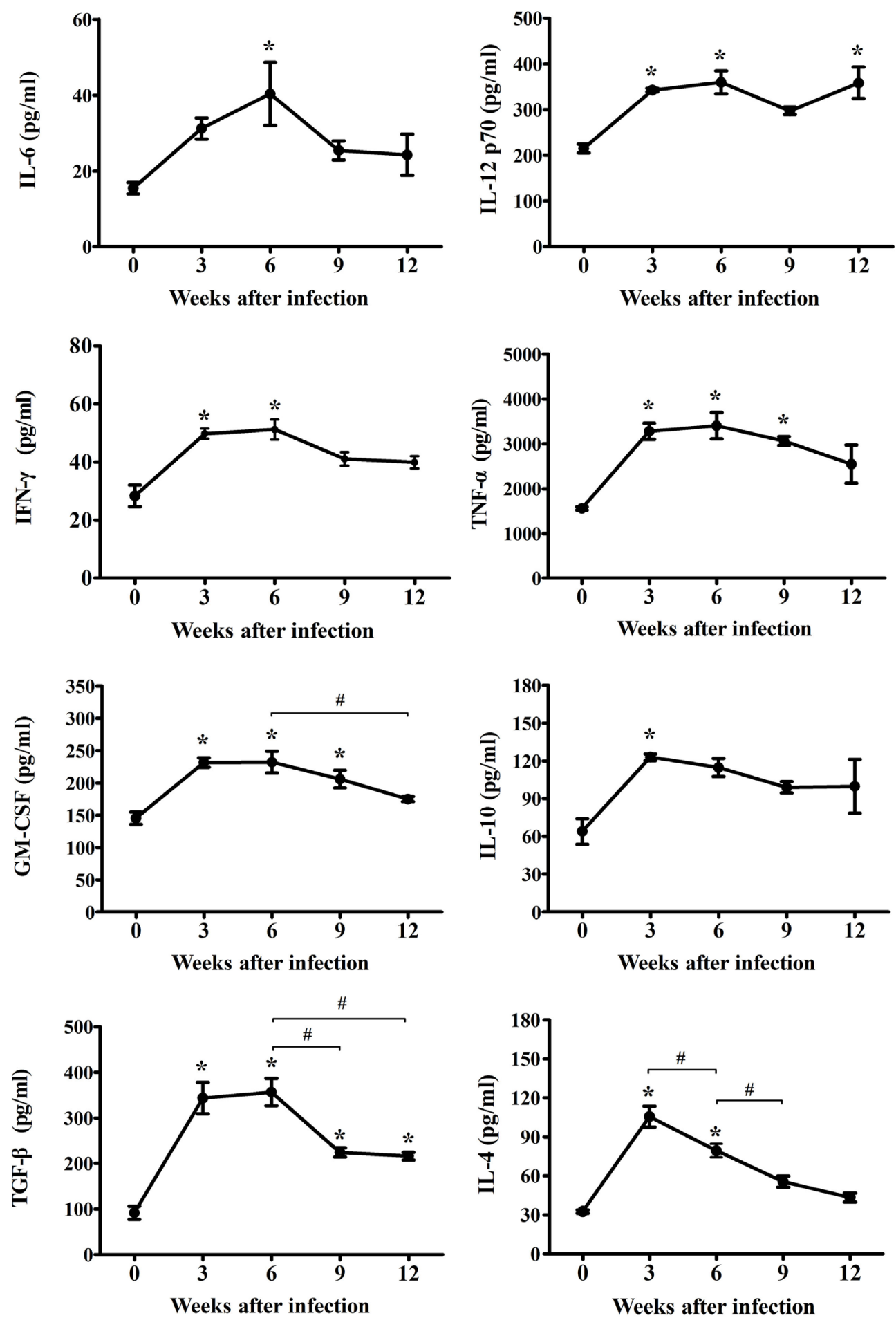

FIGURE 2 | Expression changes of cytokines in cerebral Toxoplasma gondii infection from the acute infection stage to the chronic infection stage. Brain tissues were harvested at 0, 3, 6, 9, and 12 weeks postinfection and analyzed for changes in cytokine levels due to infection. Levels of inflammatory cytokines [IL-6, IL-12p70, IFN- $\gamma$, tumor necrosis factor (TNF)- $\alpha$, and granulocyte-macrophage colony-stimulating factor (GM-CSF)] and anti-inflammatory cytokines [IL-4, IL-10, and transforming growth factor- $\beta$ (TGF- $\beta$ )]. Cytokine levels are presented as the mean \pm SD at each infection stage. ${ }^{*} p<0.05$ (one-way analysis of variance). ${ }^{*}$ indicates significant difference compared with the control. " indicates significant difference between the experimental groups. 


\section{Profiles of Inflammatory and Anti-inflammatory Cytokines in the Mouse Brain According to the Time of T. gondii Infection}

To reveal the characteristics of the inflammatory mechanisms underlying microglia activation after T. gondii infection, inflammatory, and anti-inflammatory cytokines (IL-6, IL-12p70, IFN- $\gamma$, TNF- $\alpha$, GM-CSF, IL-4, IL-10, and TGF- $\beta$ ) were examined at 0, 3, 6, 9, and 12 weeks PI. As show in Figure 2, the concentrations of inflammatory- (IL-6, IL-12p70, IFN- $\gamma$, TNF- $\alpha$, and GM-CSF) and anti-inflammatory-cytokines (IL-4, IL-10, and TGF- $\beta$ ) had gradually and statistically increased during the AI stage (3-6 weeks PI, ${ }^{\star} p<0.05$ at every time point compared, as compared to week 0 ). Furthermore, the levels of inflammatory cytokines (IL-6, IL-12p70, IFN- $\gamma$, TNF$\alpha$, and GM-CSF) had peaked at 6 weeks PI, while those of the anti-inflammatory cytokines (IL-4 and IL-10) had peaked at 3 weeks PI, suggesting that levels of the anti-inflammatory cytokines had decreased immediately after the rapid increase during the early stage of infection (Figure 2). At this time, TGF- $\beta$, which is known to be associated with neuroprotection, continued to increase until 6 weeks PI. The peak level of each cytokine is shown in Figure 2. The abundance of the inflammatory cytokines IL- 6 , IL-12p70, IFN- $\gamma$, TNF- $\alpha$, and GM-CSF had increased from baseline (week 0 ) to 6 weeks PI by 262, 167, 180,218 , and $160 \%$, respectively, whereas the abundance of the anti-inflammatory cytokines IL- 4 and IL-10 had increased by 324 and $192 \%$ at 3 -weeks PI, respectively, and that of TGF- $\beta$ had increased by $389 \%$ at 6 weeks PI. The cytokine IFN- $\gamma$, which produces a noxious cellular effect on the CNS and simultaneously activates the inflammatory cellular responses, had decreased at 6 weeks PI, whereas the levels of IL-12, which activates microglia, were sustained with no remarkable decrease during the experimental period. Moreover, levels of the cytokine TGF- $\beta$, which induces neuroprotective antiinflammatory responses, were mostly sustained during the experimental period, but had decreased slightly. The levels of the anti-inflammatory cytokines IL- 4 and IL-10 had decreased significantly during the CI stage (9-12 weeks). The results of the present study showed that levels of the inflammatory cytokines had continually increased to 6 weeks PI and were maintain at high levels even during the CI stage, although there were slight decreases, whereas levels of the anti-inflammatory cytokines had remarkably increased during the AI stage (3 weeks PI) and then decreased immediately thereafter during the CI stage. The most important finding was the significant increase and maintenance of IL- 12 and TGF- $\beta$ levels during the CI stage for 12 weeks PI (Figure $2,{ }^{\star} p<0.05$ ).

\section{Relative Cytokine mRNA Levels and Microglia Phenotype Markers for T. gondii-Infected Mouse Brain}

Changes in gene expression levels of inflammatory and antiinflammatory cytokines after $T$. gondii infection are shown in Figure 3. Gene expression levels of each cytokine at each time point of infection were compared to those of normal brain tissues (onefold at week 0 PI, a faint khaki color). Data are presented as heat maps (Figure 3), which depict the most commonly up- and downregulated transcripts from microarray analysis. The data presented in blue and yellow indicate reduced and increased cytokine expression levels. The present study analyzed gene expression patterns of inflammatory and antiinflammatory cytokines (Figure $\mathbf{3 A}$ ), as well as the microglia phenotype markers of the M1 and M2 types (Figure 3B). As compared with the anti-inflammatory cytokines (IL-4, IL-10 and TGF- $\beta$; 1.0 - to 1.6 -fold), transcript levels of the inflammatory cytokines (IL-12, IFN- $\gamma$, and TNF- $\alpha$ ) had increased by 1.5 - to 3.3 -fold, as compared to $\log _{2}$-values (onefold) of the transcript levels of control mice. Especially, IFN- $\gamma$ was remarkably increased by 3.3 -fold at 3 weeks PI and was constantly maintained at 2.2 -fold at 12 weeks PI. Likewise, the inflammatory cytokine TNF- $\alpha$ and the anti-inflammatory cytokine TGF- $\beta$ were relatively increased by 1.5 - to 1.8 -fold during the 12-week experimental period. Expression levels of the phenotype markers M1-type and M2-type were found to be polarized in the M1-type microglia (Figure 3B). Most importantly, Arg1 levels among M2-markers were consistently increased for 12 weeks PI at 1.7- to 3.4-fold, even though the increase in IFN- $\gamma$ levels was 2.2- to 3.3-fold. Further examining of the 12 polarization markers in each type of microglial cell phenotype showed that nine markers of the M1-type (IL-1 $\beta$, IL-12, TNF- $\alpha$, IFN- $\gamma$, CCL5, CXCL1, CXCL10, CD16, and CD86) and four markers of the M2-type (IL-1R $\alpha$, TGF- $\beta$, Arg1, and YM1) were consistently expressed for the entire 12-week period after infection (Figure 3B). Most notably, there was no increase in iNOS, while Arg1 was remarkably increased even if polarization and activation of M1-type microglia were distinct during the CI stage (Figure 3B).

\section{Kinetics of Microglia Activation and Phosphorylation of Stat1 in T. gondii-Infected Mouse Brain Tissues}

The above results showed that the expression levels of the inflammatory cytokines (IL-12, IFN- $\gamma$, and TNF- $\alpha$ ) had continuously increased during the CI stage. Among these cytokines, mRNA and protein levels of IL-12, a key cytokine of microglial cells activation, and IFN- $\gamma$, a key cytokine of Th1-helper T-cell activation, were maintained steadily with high levels during the CI stage (Figures 2 and $\mathbf{3}$ ). To comprehensively link changes between gene and protein expression levels, brain tissues were immunohistologically stained with Iba-1- and phosphorylated STAT-1 (pStat1) antibodies for microglial activation and to determine whether these molecules participate in the IFN- $\gamma$-mediated pathway, respectively (Figures 4A,B). Because IFN- $\gamma$ plays a role in adaptive cellular immunity against $T$. gondii infection and STAT-1 is important for intracellular downregulation of IFN- $\gamma$, the increase in pStat1-stained cells in the brain indicates an increase in IFN- $\gamma$-mediated Th1-T-cell immune responses. However, pStat1-stained cells were observed around the cyst (red arrow) over a relatively short period of time at 6 weeks PI and, thereafter, were undetectably in the brain tissues (Figure 4B). At this time, the proportion of microglial cells had remarkably increased around the T. gondii cysts (red arrow) during the AI stage and 


\section{A Cytokine}

$-3$

$+3$
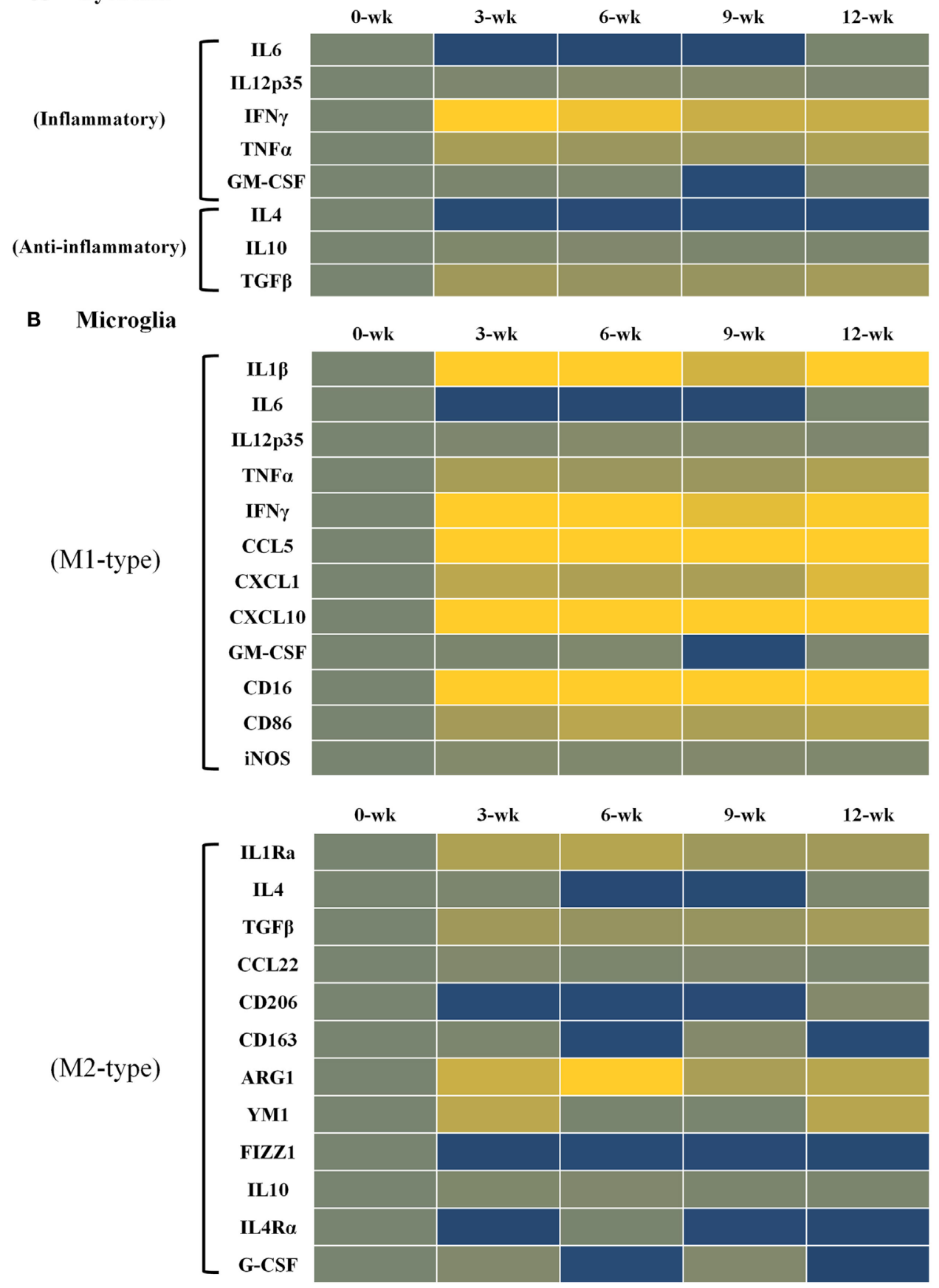

FIGURE 3 | Heat maps representing cytokine and chemokine concentrations in the inflammatory immune response and microglia phenotypes. Transcript levels of inflammatory and anti-inflammatory cytokines as well as microglia phenotype markers were measured in Toxoplasma gondii-infected mouse brains using microarray analysis. Each row of the heat map represents cytokines related with inflammatory and anti-inflammatory responses (A) as well as microglia phenotype markers related with the M1-type and M2-type (B). Each column represents infection times from week 0 to 12 postinfection. The color scale corresponds to the relative expression of the cytokine for the minimum $(-3)$ and maximum $(+3)$ of all values.

was activated continuously during the CI stage (Figure 4A). This activation of microglial cells can be explained by morphological characteristics, such as hyper-ramification and long, thin processes extending from the cell body into the surrounding milieu (Figure 4A, colored in brown). The increase in activated microglia was greatest at 6-9 weeks PI and then had decreased 


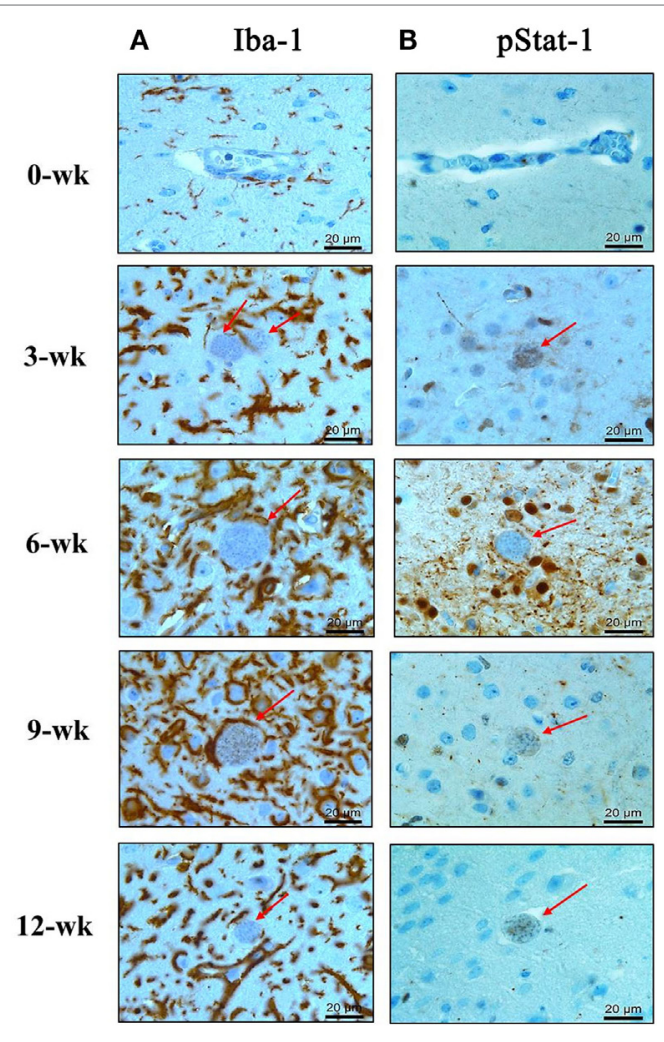

FIGURE 4 | Phosphorylation of Stat1 (pStat1) and lba-1-stained microglia infiltrated and activated around Toxoplasma gondii cysts. T. gondii-infected mouse brains were harvested at $0,3,6,9$, and 12 weeks postinfection, embedded in paraffin, and immunostained with Iba-1- (A) and phosphorylated Stat-1 antibodies (B). T. gondii cysts (arrow). Activated microglia [(A) brown color]. pStat1 immunoreactivity [(B) brown color]. Magnification, 400x. Scale bar $=20 \mu \mathrm{m}$.

slightly at 12 weeks PI (CI stage). These results strongly suggest microglia are activated throughout the infection period, whereas STAT1 phosphorylation was limited and no longer maintained during the CI stage.

\section{Effects of TLA (RH- or ME-TLA) on the Polarization and Activation of Microglial Cells}

Infection immunity in T. gondii-infected brain tissues can be explained by the immune characteristics regulated by the host-parasite relationship. Infectious immunity shown in the present study was characterized by an increase in microglial cells and the limited IFN- $\boldsymbol{\gamma}$-mediated immune response. However, it remains unclear whether these immune responses were induced by immune regulation via the host-parasite relationship or by immunological triggering of T. gondii antigens as a PAMP. For this purpose, TLAs were prepared from RH- or ME49 tachyzoites to investigate the effects of TLA on the activation and polarization of microglia, which were further compared with transcript levels in the brain (Figures 5A,B). TLAs contain many PAMPs and stimulate toll-like receptor (TLR)-based immune responses. Moreover, because T. gondii strains ( $\mathrm{RH}$ and ME49) have different modes of infectious immunity, the comparison of $T$. gondii antigens (RH-TLA or ME-TLA) on microglia activation is important to evaluate difference in immune responses between the AI and CI stages. As shown by the results, the MFI (FACS analysis) of the activation markers of BV-2 microglial cells [i.e., major histocompatibility complex II (MHCII), CD40, CD80, and CD86] had increased in response to IFN- $\gamma$ stimulation (Figure 5A). Such an increase in MFI indicates an increase in target molecules in FACS. IFN- $\gamma$ stimulation of BV-2 cells had increased the expression levels of these activation markers, which was accelerated by the presence of TLA (Figure 5A). At this time, ME-TLA had a greater effect on the increase in activation markers than RH-TLA. In contrast, expression of the M2 polarization marker CD206 was lower in ME-TLA than RH-TLA (Figure 5A). These results are consistent with the microarray results (Figure 5B). Subunit transcripts of MHC class II molecules (H2-Eb1, -Aa, and -Ab1), Cd40, Cd80, and Cd86 were increased (16). In contrast, $C d 206$ was decreased, as compared to the control (khaki) (Figure 5B). The M1 markers Cd40, H2$E B 1(-A a,-A b 1)(\mathrm{MHCII})$, and $C d 86$ (B7-2) were upregulated in activated microglial cell and acted as co-stimulatory molecules for further T- and B-cell immune responses. Both mRNA and protein levels of the representative M2 marker CD206 were decreased simultaneously in cells infected with $T$. gondii strain ME49, as well as by antigen treatment (ME-TLA) as PAMPs. Accordingly, our results showed that both TLA treatment and T. gondii infection induced the activation and M1 polarization of microglial cells compared with the control (no-treatment of IFN- $\gamma$ and lysate antigens in vitro study as well as 0 -week PI in vivo study).

\section{Changes in Transcripts for T-Cell Differentiation- and T-Cell Exhaustion-Markers in the Brain during T. gondii Infection}

CI of T. gondii in the brain may induce T-cell exhaustion and dysfunction. The results of this study showed a decrease in the IFN- $\boldsymbol{\gamma}$-mediated immune response during CI even if microglial cell activation was maintained. To arrive at a possible explanation, markers of T-cell differentiation and dysfunction were examined, which showed that Th1 cell-specific transcription factor TBX21 that controls the expression of IFN- $\gamma$ was mainly increased during CI, as compared to the Th2 cell-secreted cytokines Gata3 and Foxp3, which promote the functions of regulatory T cells (Figure 6A). Nevertheless, markers of T-cell exhaustion, including Tim3 and Lag3, were also increased simultaneously from the AI stage at 3 weeks PI (Figure 6B). Tim 3 and Lag3 are known to negatively regulate T-cell proliferation, homeostasis, and Th1 immunity, and act as immune checkpoints. This result means that T. gondii infection actively influences the induction of the host immune response against infection. In other words, although these findings were limited to transcript profiles, T. gondii infection of the brain simultaneously induces both T-cell differentiation and exhaustion from the AI stage at 3 weeks PI, and maintains the immune environment during the entire the CI stage (Figure 6). 

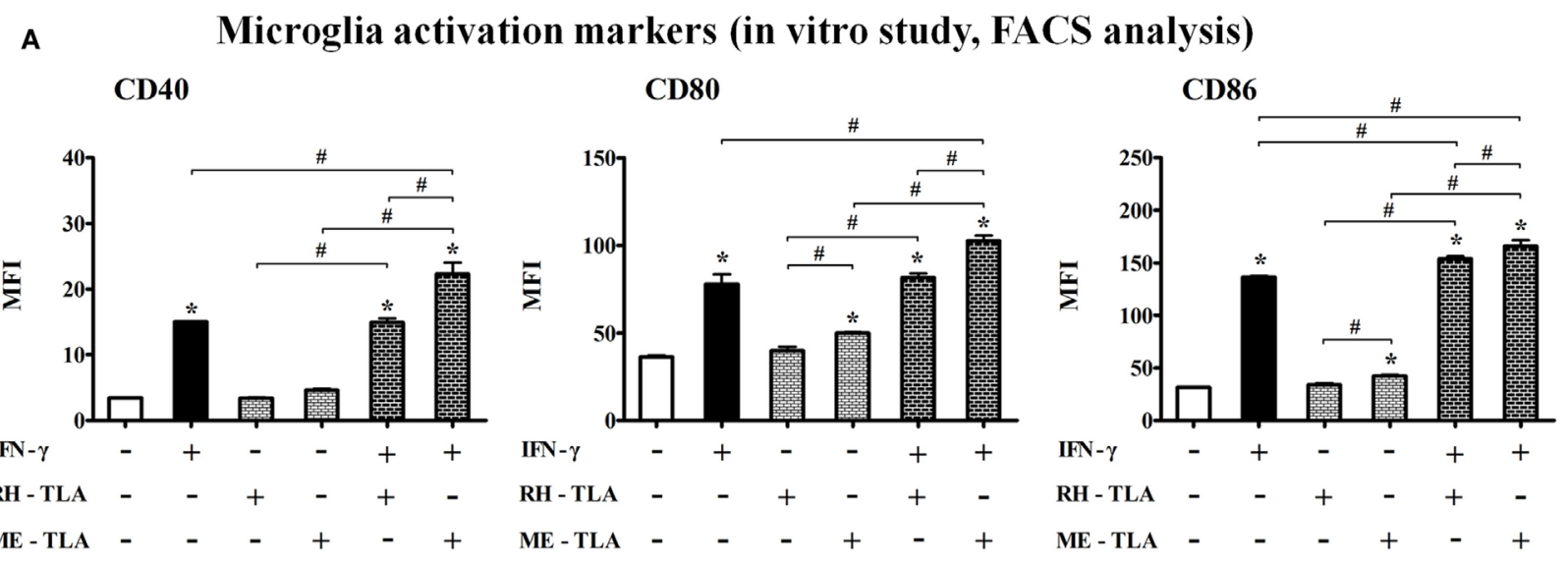

MHC II
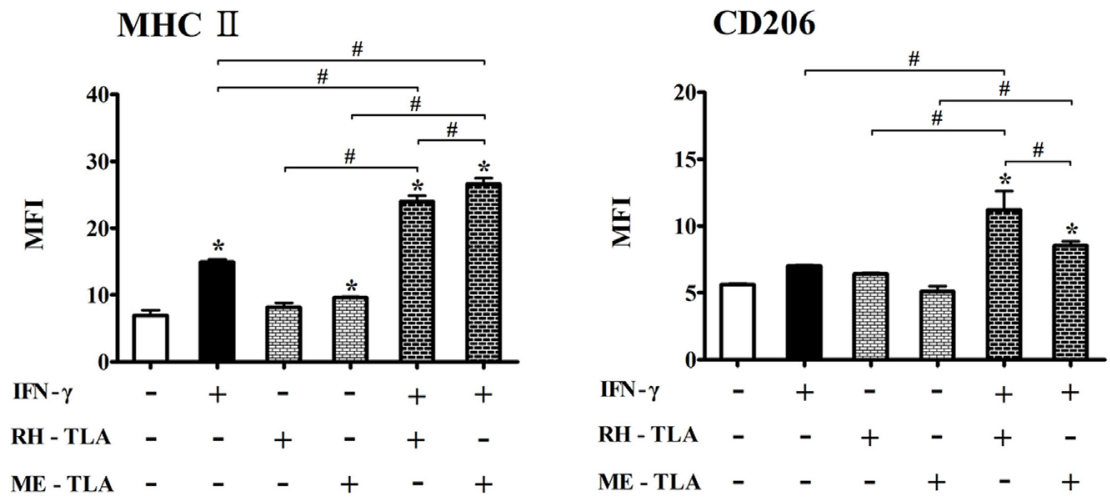

B

Microglia activation markers (in vivo study, Transcripts in the brain)

0-wk

3-wk

6-wk

9-wk

12-wk

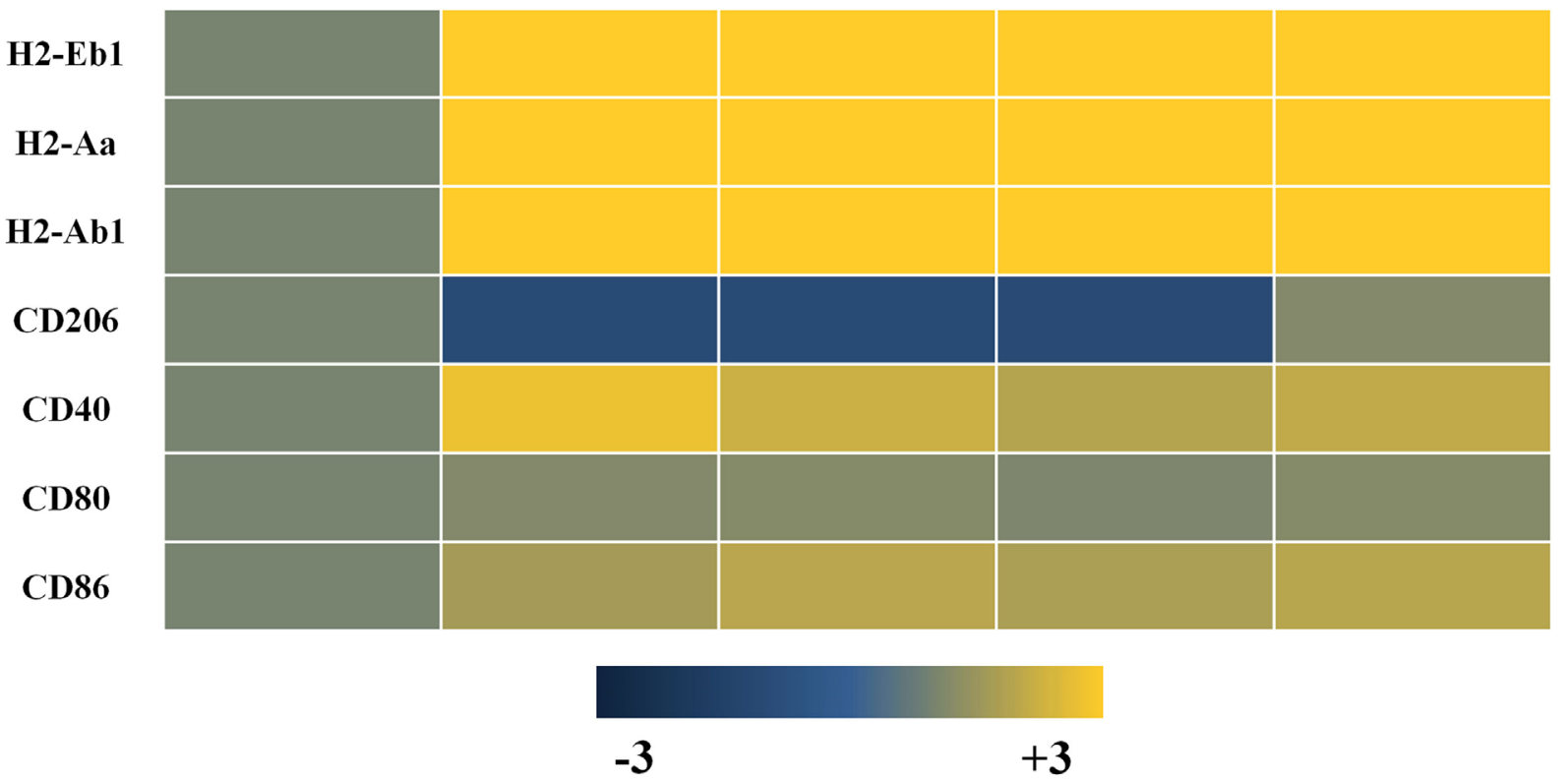

FIGURE 5 | Microglia activation in Toxoplasma gondii-infected brain and BV-2 cells stimulated with T. gondii lysate antigens (TLAs) (RH-TLA and ME-TLA). Expression levels of major histocompatibility complex II antigens (H2-Eb1, H2-Aa, and H2-Ab1), CD40, and CD86 by FACS analysis. (A) Heat map expression of cell surface markers related with microglia activation in $T$. gondii-infected brains, (B) results are expressed as the mean \pm SD of the mean fluorescence intensity. ${ }^{*} p<0.05$ (one-way analysis of variance). * indicates significant difference compared with the control. * indicates significant difference between the experimental groups. 


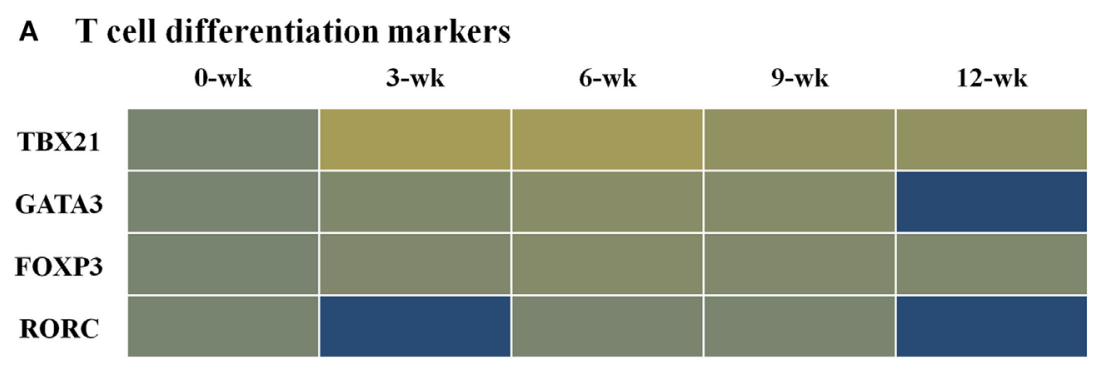

B $\mathbf{T}$ cell exhaustion markers

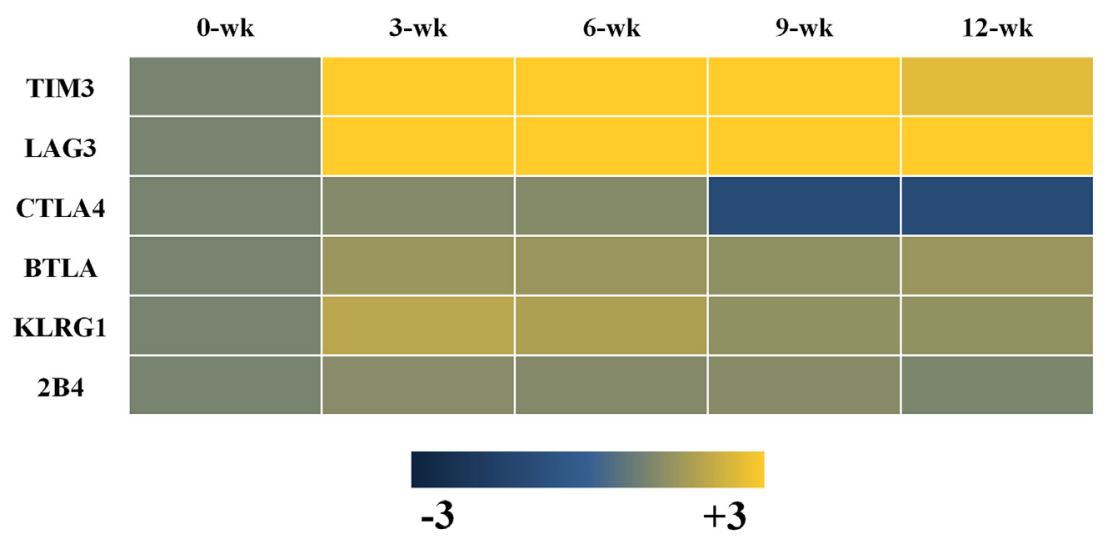

FIGURE 6 | Changes in T-cell differentiation (A) and exhaustion (B) markers in Toxoplasma gondii-infected brain tissues. Expression values represent the intensity of gene expression varying from -3 to +3 colored with blue or yellow.

\section{Changes in mRNA Levels of Immune Effector and Checkpoint Molecules in T. gondii-Infected Brain Tissues}

To investigate the expression of effector molecules associated with both inflammatory and anti-inflammatory responses, changes in transcripts and quantitative gene levels of Socs1, iNos, and Arg1 during the 12-week experimental period were compared to those at baseline (week 0 PI) (Figure 7A). Transcript levels of Socs 1 , which is thought to be an important immune regulatory factor in this study, was increased by 1.5 -fold at 3 -weeks PI and maintained during the CI stage (Figure 7A). Likewise, quantitative gene expression levels of Socs 1 was the highest at 3 weeks PI (2.63-fold) with statistical significance and, thereafter, had slightly decreased during the CI stage. Similarly, the results of western blot analysis showed an increase in SOCS1 expression during the infection period. In contrast, there was no significant increase in iNOS mRNA levels, and quantitative gene expression levels of iNOS/Arg1 were decreased even during the AI stage ( 3 and 6 weeks PI). This result is also supported by transcript level of Arg1 (2.2- and 3.4-fold at 3 and 6 weeks PI, respectively) (Figure 7A). Expression levels of the of the immune inhibitory checkpoint molecules PD-1, PD-L1, and PD-L2, as related with T-cell dysfunction, were examined at the transcript level and by FACS analysis. As shown in Figure 7B, transcript levels of PD-1, an inhibitory receptor of T cells, were slightly increased by 1.1 - to 1.4-fold at week 12 PI, whereas levels of PD-L1 were remarkably increased by 19.5-, 21.4-, 18.9-, and 23.0-fold at 3, 6, 9, and 12 weeks PI, respectively. This result was also strongly supported by the FACS results expressed as the MFI, suggesting strong activation of the M1-type microglia. When RH- or ME-TLA as PAMPs of T. gondii stimulate BV-2 microglial cells, the increase in the MFI of PD-L1 was greater than that of PD-L2 and the degree of increase was greater via stimulation of ME-TLA than RH-TLA. Accordingly, it is expected that the immune-triggering effect of T. gondii strain ME49 during CI seems to limit the expression of inflammatory effector molecules such as iNOS, even with activation of innate immunity by microglial cells.

\section{Effects of T. gondii Antigens (RH- and ME-TLA) on Nitrite and Urea Production according to Microglia Polarization}

For 12 weeks PI, iNOS production was decreased and Arg1 production was increased. As well, when BV-2 cells were treated with T. gondii antigens as a PAMPs, expression levels of the activation markers were increased. In this regard, an aim of this study was to investigate whether treatment of BV-2 microglial cells with T. gondii antigens (RH-TLA or ME-TLA) can induce changes in $\mathrm{NO}$ and urea production, as with T. gondii-infected brain tissues. In general, nitrite production is mainly increased as a result of M1-type activation, whereas urea production is increased by M2-type microglia activation. The results of the present study showed that the production of nitrite was increased by stimulation 

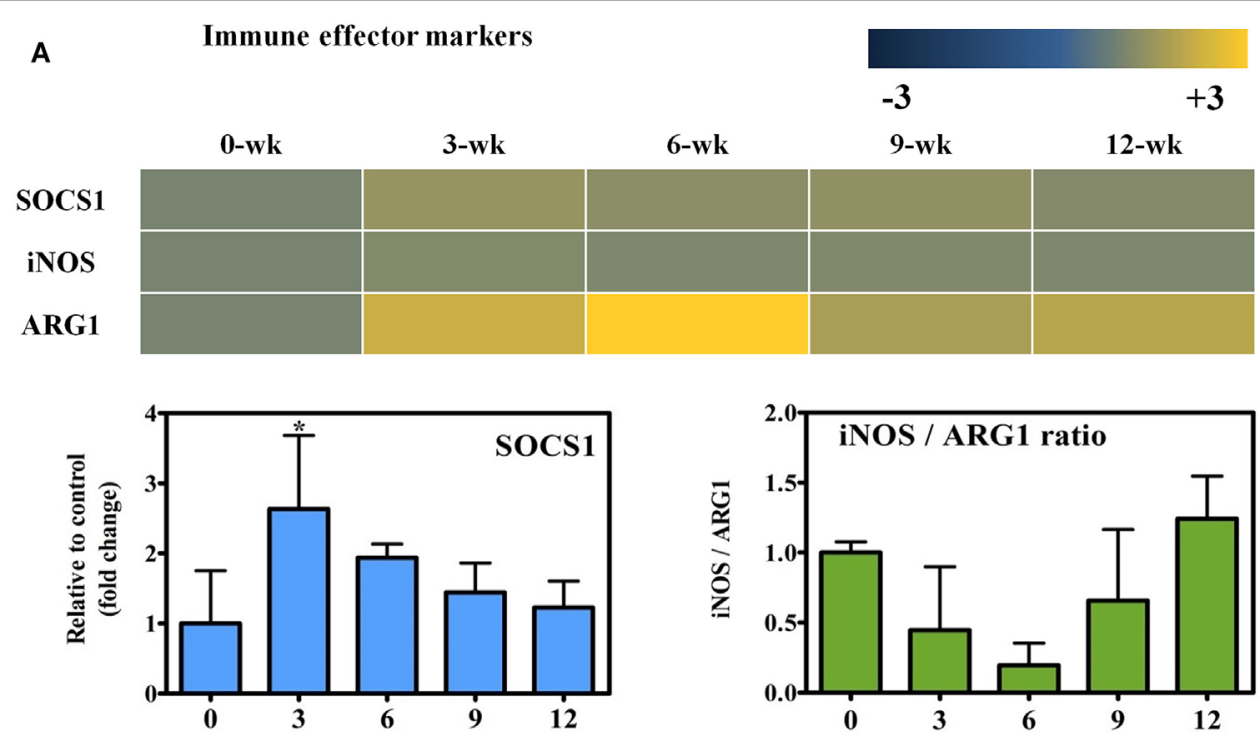

Weeks after infection

SOCS1

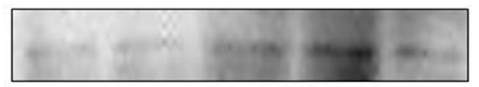

$\beta$-actin

Weeks after infection

B

Immune checkpoint molecules
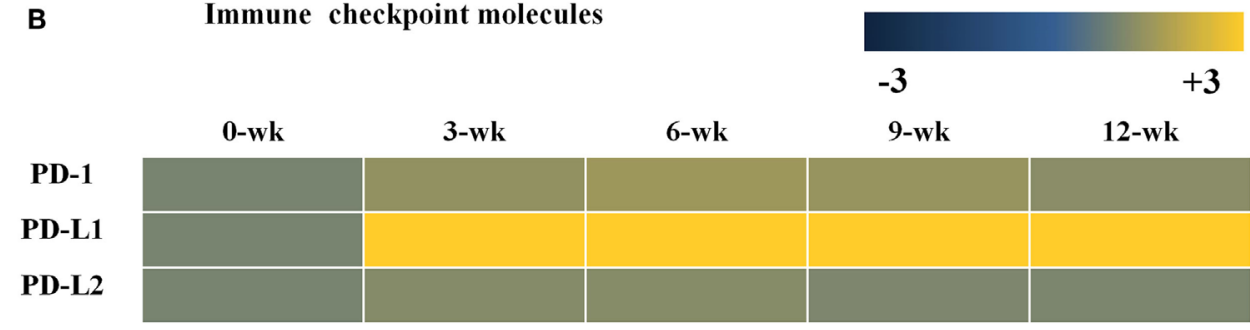

PD - L1

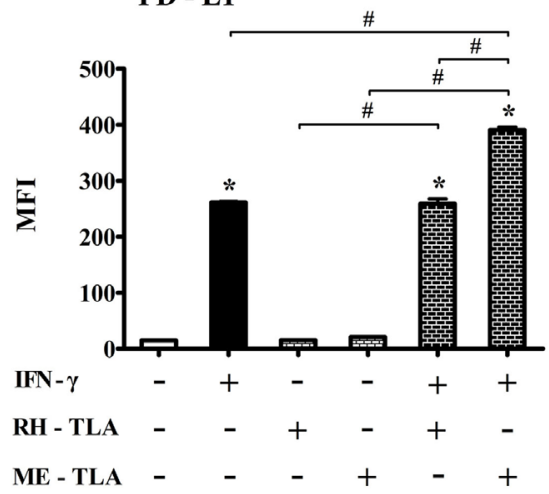

PD - L2

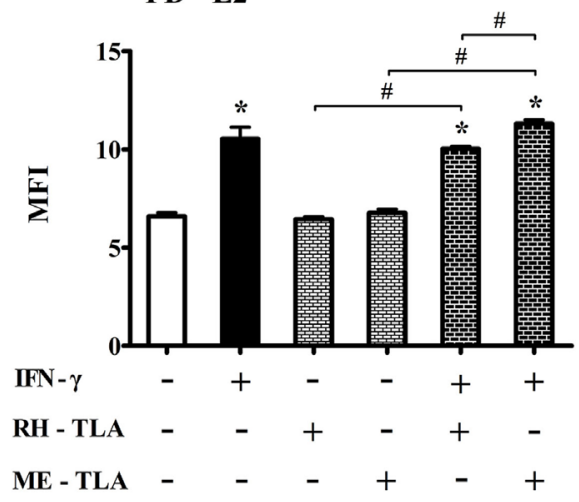

FIGURE 7 | Changes in immune effector and checkpoint molecules. Transcripts of immune effector markers [nitric oxide synthase (iNos) and Arg1] (A), immune control marker (Socs1) (A) and immune checkpoint markers (PD-1, PD-L1 and PD-L2) (B). RT-PCR results (SOCS1 and the iNos/Arg1 ratio) and western blot (SOCS1 and $\beta$-actin) (A). Transcript expressions(PD-1, PD-L1, and PD-L2) and FACS analysis (PD-L1 and PD-L2) (B). Results are expressed as the mean \pm SD of the mean fluorescence intensity (MFI). ${ }^{*} p<0.05$ (one-way analysis of variance). * indicates significant difference compared with the control. ${ }^{*}$ indicates significant difference between the experimental groups.

of IFN- $\gamma$ alone $(9.5 \pm 0.3 \mu \mathrm{M})$ or IFN- $\gamma$ with $T$. gondii antigen $(6.6 \pm 0.2 \mu \mathrm{M}$ with RH-TLA and $17.1 \pm 0.7 \mu \mathrm{M}$ with ME-TLA), as compared to the control $(4.3 \pm 0.4 \mu \mathrm{M})$ (Figure 8A). At this time, nitrite concentration had significantly increased via stimulation with ME-TLA, as compared to RH-TLA, suggesting that ME-TLA itself is more preferable for the induction of M1-type 

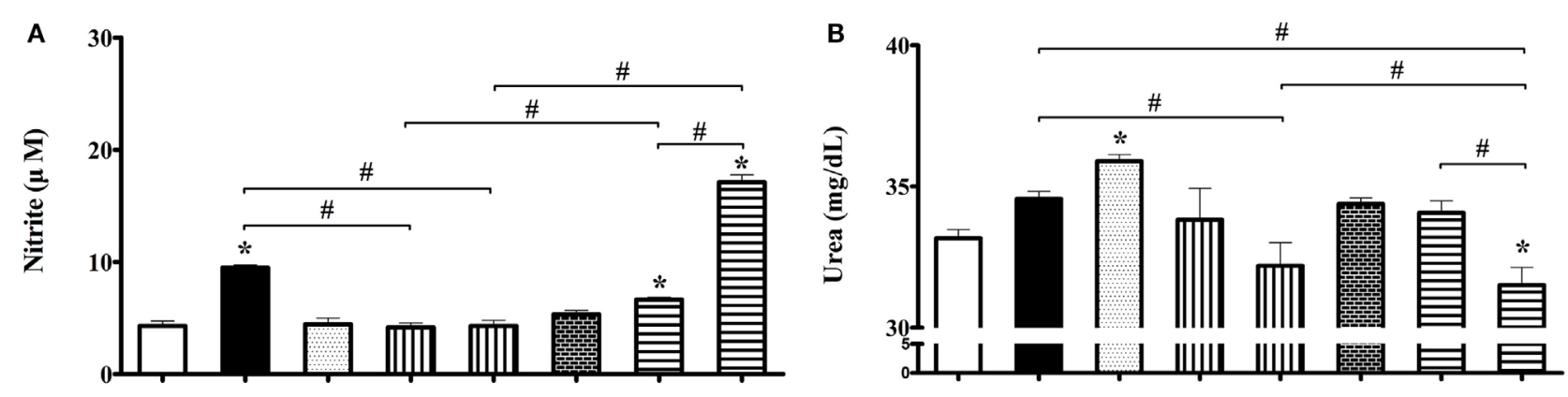

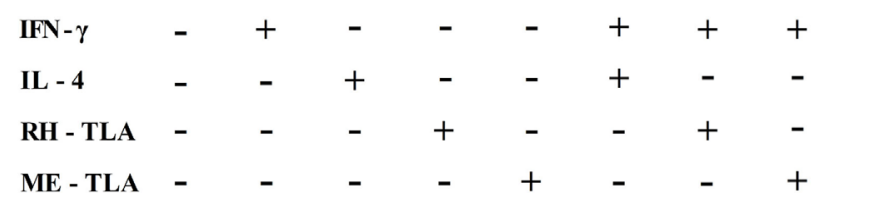

$\begin{array}{lllllllll}\mathrm{IFN}-\gamma & - & + & - & - & - & + & + & + \\ \mathrm{IL}-4 & - & - & + & - & - & + & - & - \\ \mathrm{RH}-\mathrm{TLA} & - & - & - & + & - & - & + & - \\ \mathrm{ME}-\mathrm{TLA} & - & - & - & - & + & - & - & +\end{array}$

FIGURE 8 | Nitrite and urea production in BV-2 microglia stimulated with recombinant cytokines affecting microglia polarization (IFN- $\gamma$ and IL-4) or Toxoplasma gondii antigens $\left(\mathrm{RH}-\mathrm{TLA}\right.$ or ME-TLA). Nitrite $(\mu \mathrm{M})(\mathbf{A})$ and urea $(\mathrm{mg} / \mathrm{dL})$ concentrations $\mathbf{( B )}$. Data are presented as the mean $\pm \mathrm{SD}$. ${ }^{*} p<0.05(\mathrm{one}-\mathrm{way}$ analysis of variance). * indicates significant difference compared with the control. \# indicates significant difference between the experimental groups.

microglial activation $\left({ }^{\star} p<0.05\right)$. In contrast, urea concentration was increased significantly by stimulation of IL-4, as compared to the control $(36.0 \pm 0.2$ vs. $33.2 \pm 0.3$, respectively) (Figure $8 B$ ) As well, urea production was more decreased by ME-TLA than RH-TLA ( $32.2 \pm 0.8$ vs. $33.8 \pm 1.1 \mathrm{mg} / \mathrm{dL}$, respectively). Notably, treatment with IFN- $\gamma+$ ME-TLA had decreased urea production even further than with treatment of IFN- $\gamma+$ RH-TLA $(31.5 \pm 0.6$ and $34.1 \pm 0.4$, respectively, $\left.{ }^{\star} p<0.05\right)$. These results clearly show that treatment of BV-2 cells with ME-TLA induced M1 polarization and M1-activation, which subsequently remarkably increased nitrite production and decreased urea production, suggesting that the immune induction of ME-TLA acts as a PAMP. However, this result is in contrast to the transcript results of the T. gondii-infected brain tissues. To clarify this difference, infection of BV-2 cells was performed in vitro (Figure 9).

\section{Effects of In Vitro T. gondii Infection (RH- and ME-Strain) on mRNA Expression (iNOS, Cox-2, and Arg1) and Nitrite Production}

To elucidate the differences in inflammatory responses and NO production between $T$. gondii infection in the brain and in vitro experiments with the T. gondii antigens (RH-TLA and ME-TLA), BV-2 cells were infected with T. gondii tachyzoites in vitro. As shown in Figure 9, the expression levels of the inflammatory response markers iNos and Cox-2 were significantly decreased after $T$. gondii infection in vitro, whereas that of Arg1 was significantly increased. Notably, Arg1 expression levels at $24 \mathrm{~h}$ after in vitro $\mathrm{RH}$ and ME49 infection were increased by $2.0 \pm 0.3$ - and $2.9 \pm 0.1$-fold, respectively, as compared to control $\left({ }^{*} p<0.05\right)$. Likewise, Arg1 induction was significantly greater after infection with strain ME49, as compared to strain RH. At this time, nitrite production was the highest with treatment of IFN- $\gamma$ alone $(39.6 \pm 2.0 \mu \mathrm{M})$ and decreased significantly with IFN- $\gamma+T$. gondii infection (RH and ME49) $(28.9 \pm 1.4$ and $24.0 \pm 0.9 \mu \mathrm{M}$, respectively, $\left.{ }^{*} p<0.05\right)$. As compared to IFN- $\gamma$ alone, nitrite levels were $27 \%$ lower with IFN- $\gamma+T$. gondii strain $\mathrm{RH}$ infection and 39.4\% lower with IFN- $\gamma+$ T. gondii strain ME49 infection. Accordingly, these results emphasize that in vitro T. gondii infection induces polarization of microglial cells into the M1-type. However, simultaneous inhibition of harmful inflammatory factors, such as NO, had increased Arg1 expression for further immune regulation. This is an important result showing that in vivo and in vitro infection was consistent. In other words, TLAs acting as PAMPs had strongly induced polarization of M1-type microglial cells and inflammatory responses, while infection of T. gondii (in vivo and in vitro) induced polarization of M1-type microglial cells. However, it simultaneously inhibited a detrimental inflammatory response, such as NO production, via Arg1 induction. This host-parasite relationship seems to be a strategy of $T$. gondii to maintain a CI in the brain.

\section{DISCUSSION}

The results of our previous study demonstrated that T. gondii infection decreased the neurodegeneration in a $\operatorname{Tg} 2576$ mouse model of Alzheimer's disease (8). In that study, T. gondii infection of the brain inhibited neuronal degeneration, as well as learning and memory impairments in Tg2576 mice. As a possible reason, we proposed that the increase in the expression levels of the antiinflammatory cytokines IL-10 and TGF- $\beta$, as well as decreased NO production had favorable effects of $T$. gondii infection and the pathogenesis and progression of Alzheimer's disease in mice (8). However, because inflammatory responses induced by IFN- $\gamma$ and NO are essential for the control of T. gondii infection, NO regulation in the CNS is very important to control both parasitic proliferation and damage to the host tissues. As an extension of the above results, the aim of the present study was to address the progression of infection immunity to identify the appropriate timing to induce an anti-inflammatory response during CI. In 


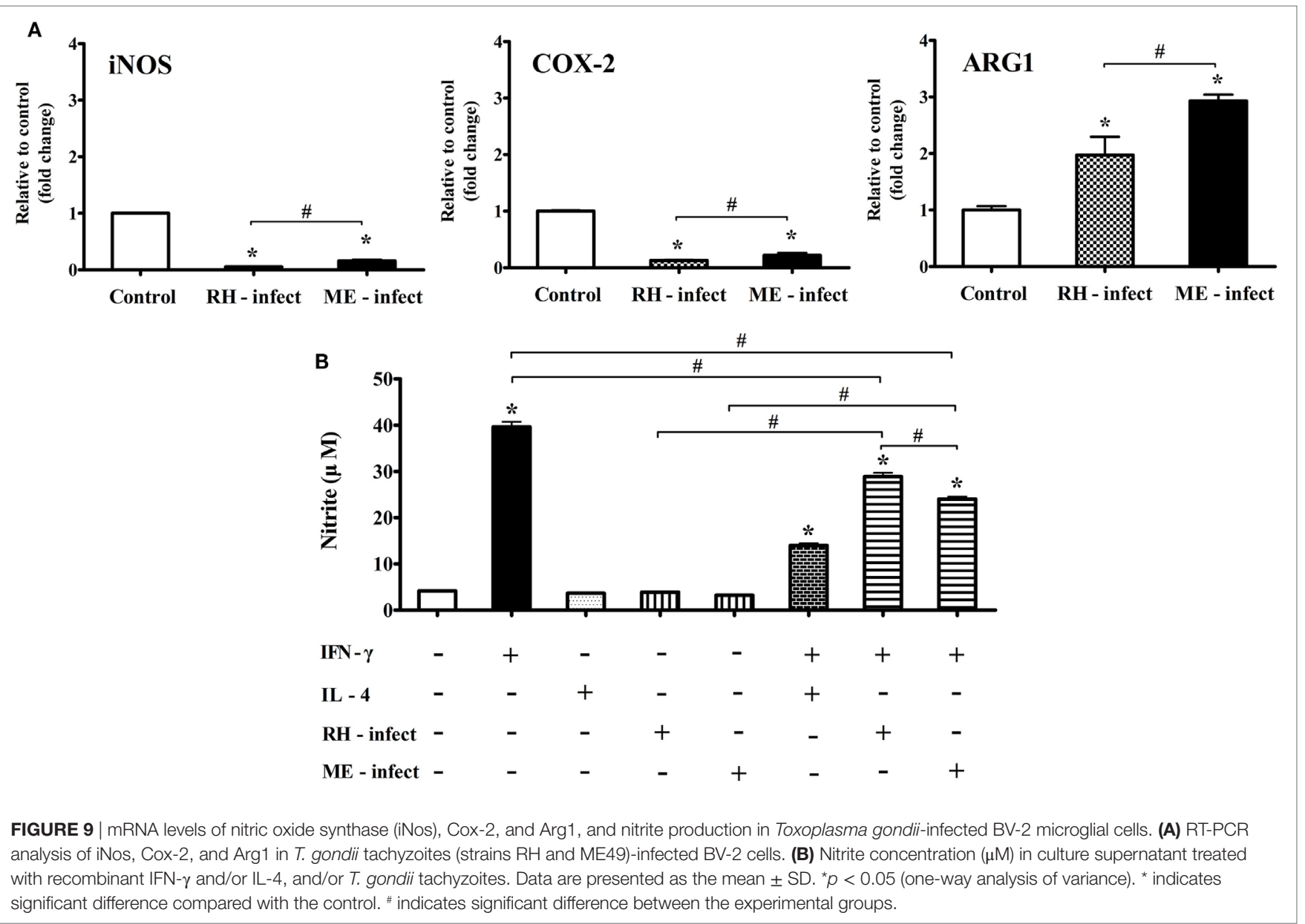

particular, because T. gondii is a pathogen that can lead to CI of the host, as well as induce encephalitis and neurodegeneration, it is very important to understand the infection immunity related with microglia polarization and harmful inflammatory responses.

Encephalitis is an important symptom of T. gondii infection, although the neuronal degeneration associated with neuroinflammation is not a common finding because immune modulation by $T$. gondii infection has been the focus of numerous previous studies $(1-5,8,9)$. In the present study, neuronal cell death caused by $T$. gondii infection in the DG region of the hippocampus was increased in the AI stage at 3-6 weeks PI, which then decreased in the CI stage accompanied with slight decreases in the expression levels of the inflammatory cytokines IL- 6 , IFN- $\gamma$, TNF- $\alpha$, and GM-CSF. Even in this case, the Iba-1 staining intensity of microglial cells was not significantly decreased during the entire 12-week experimental period. In fact, the microarray results of the T. gondii-infected brain and FACS analysis of in vitro cultured BV-2 microglial cells with lysate antigens (RH-TLA and ME-TLA) prepared from tachyzoites of $T$. gondii strains $\mathrm{RH}$ and ME49, showed M1 polarization of microglial cells both in vivo and in vitro. However, high levels of the inflammatory and anti-inflammatory cytokines remained during the 12-week experimental, even though the levels had slightly decreased from 6 weeks PI. Thus, it is necessary to consider why neuronal cell death was highest at 3 weeks PI and then decreased. Clearly, T. gondii infection induces a mixed immune response characterized by an increase in the expression levels of inflammatory cytokines and the activation and proliferation of microglial cells, as well as increases in the expression levels of the anti-inflammatory cytokines and Arg1 and decreased expression of iNOS and COX-2 accompanied with NO production.

The strategy of $T$. gondii intracellular parasitism involves the manipulation of an excessive response of immune cells and alterations in macrophage phenotypes to suppress inflammatory response and reduce damage to the host. According to the results of an earlier study, T. gondii infection is generally controlled by a strong Th1-type immune response via the cytokines IL-12p70, TNF- $\alpha$, and IFN- $\gamma$. However, the recruited monocyte population is converted to an anti-inflammatory phenotype in order to restrain excessive immune responses during infection of $T$. gondii strain RH $(3-6,12,14,21,22)$. On the other hand, the time of infection for the change in microglial cells and inflammatory responses as a cause of CI of the brain infected with strain ME49 it is not well defined. Some studies have provided important clues to prevent excessive activation of macrophages after $T$. gondii infection $(3,14)$. For example, Toxoplasma rhoptry and dense granule proteins play roles in the modulation of pro-inflammatory responses 
through the regulation in IFN- $\gamma$ and NF-kB by GRA15 in addition to STAT3/6 activation by $\operatorname{ROP} 16(3,14)$. ROP16 induces STAT1 tyrosine phosphorylation as well as Socs 1 via STAT3 or 6 (23). As a result, the authors insisted that the infected hosts are able to induce a proper resistance against $T$. gondii infection as well as protection against excessive inflammatory immunity $(3,14,23)$. However, these studies were limited by the failure to explain the promotion of immune modulation during T. gondii infection because the experiments employed Raw264.7, J447, DC2.4, or human foreskin fibroblast cells for in vitro T. gondii infection and T. gondii-infected peritoneal exudate cells for in vivo infection $(3,14)$. So, these studies do not reflect the entire infection process. On the contrary, the present study addressed the changes in the infection immunity during the CI stage. For example, the immune regulation for the control of parasitic proliferation without inducing harmful immunopathological events in the brain. Moreover, the present study provides some evidence of the immune regulation against $T$. gondii infection and protective immunity to prevent damage to host cells by an excessive inflammatory response.

The IFN- $\gamma$-mediated type 1 immune response with upregulation of the anti-parasitic factor NO is required to prevent the reactivation of $T$. gondii and $\mathrm{TE}$ in the brain (1). In the brain, microglia and infiltrating CD4 and CD8 T cells are the primary sources of IFN- $\gamma$, a cytokine leading noxious cellular effects in the CNS $(1,4)$. On the contrary, our earlier research also highlighted the importance of TGF- $\beta$ in neuroprotection after T. gondii infection (8). In addition, the results of the present study suggest the importance of the interactions of various immunological factors between protective immunity and immunopathological effects by tracking of the immune response during the CI stage. First of all, changes in the immune response after $T$. gondii infection can be confirmed by microarray analysis of mRNA expression patterns, which can also predict the immunological phenotype. In the present study, there were increases in the mRNA levels of the T-cell exhaustion markers TIM3, LAG3, and KLRG1 without distinct increases in the mRNA levels of the T-cell differentiation markers TBX21, GATA3, FOXP3, and RORC during the AI stage, even though microglia activation was maintained in the M1-type. T-cell exhaustion markers are highly expressed by chronic antigen stimulation, but yet diminish the effector function of CD8 T cells (24). Accordingly, these results suggest that the inhibition of immune extension of adaptive T-cell immunity can accelerate neuronal cell death by expanding inflammatory cellular responses, although it is helpful for protective immunity of the host against T. gondii infection. Actually, pStat1, which is activated by IFN- $\gamma$ and induces iNOS (25), was remarkably decreased at 6 weeks PI although the increase in IFN- $\gamma$ expression was maintained for 12 weeks PI. Although IFN- $\gamma$-mediated activation of macrophages is critical for resistance against AI with T. gondii, T. gondii infection inhibits STAT1 transcriptional activity to allow the parasite to establish a CI $(5,23)$. Additionally, SOCS proteins, which can downregulate phosphorylation of JAK and STAT1, are actually induced by $T$. gondii infection (26). To understand the importance of microglia activation and further T-cell immune responses against $T$. gondii infection, transcript data of markers related to microglial cell polarization and T-cell-mediated adaptive immune responses were investigated. In particular, PD-L1 (B7-H1) was highly expressed in inflammatory macrophages, while PD-L2 (B7-DC) can be induced by alternative activation via IL-4 (27). Furthermore, PD-L1 might play a role in the downregulation of activated T cells and the expression of PD-L1 in a non-Stat1-dependent manner (27). Likewise, our results showed that PD-L1 expression was increased, as compared to PD-L2, at both transcript levels in vivo and in vitro ME-TLA stimulation (19.0-23.0- and 1.1-1.2-fold changes, respectively). At this time, M1-type activation of microglia and increases in the expression levels of the inflammatory cytokines IFN- $\gamma$, IL-12, and TNF- $\alpha$ were observed. However, these changes did not result in an increase in Stat-1 phosphorylation. This result provides a subtle balance for the control of parasitic proliferation and simultaneous inhibition of the increase in inflammatory cellular immune responses, which induce tissue damage during $\mathrm{CI}$ by altering the mRNA expression levels of PD-L1 and PD-1 because PD-1/PD-L1 interactions are required for the maturation of microglial cells and expansion of protective T-cell responses in T. gondii infection. However, excessive PD-1mediated CD8 T-cell dysfunction plays a central role in immune tolerance, as well as $T$. gondii differentiation and reactivation (28-30). This is an important mechanism for the control of parasite expansion, even with the inhibition of iNOS, as in the present study.

The induction of iNOS, a M1-marker that leads to NO production, is generally elevated in the AI stage of $T$. gondii infection and in M1-like microglia $(26,31)$. Surprisingly, the results of the present showed that ME-TLA, a lysate antigen of T. gondii strain ME49, strongly induces the polarization of M1-type microglial cells and NO production. In other words, ME-TLA treatment of BV-2 microglial cells induced greater M1 polarization and NO production than treatment with IFN- $\gamma$. Simultaneously, urea production was decreased by treatment with the T. gondii antigens (RH and ME49) in vitro. TLA includes a variety of antigen pools, including TLR-based immune-triggering PAMPs (32). When soluble parasite TLA, as in the present study, stimulates dendritic cells and macrophages, the immune response resulted in TLR-signaling dependent production of IL-12 and NO (32). Accordingly, it is evident that the treatment of TLA as a PAMPs and parasite infection induce different immune triggering both in vivo and in vitro. To confirm this finding, $\mathrm{NO}$ production was compared between an in vitro infection model using $T$. gondii tachyzoites (strains RH and ME49) and an in vitro model using BV-2 microglial cell culture with TLA. As expected, the results were consistent with the in vivo infection study. NO production was decreased and mRNA expression levels of iNOS and COX-2 were lowered, while mRNA expression of Arg1 was increased by 2.9-fold. Based on these results, the authors insist that the hostparasite relationship in the regulation parasite control and host tissue damage by an excessive inflammatory response induces the control of effector molecules, which differs from the immunetriggering effect of TLA shown by the in vitro stimulation study. As a possible mechanism, increases in transcripts of SOCS and Arg1 during the CI stage are focused because of their immune regulatory functions. 
Suppressor of cytokines signaling 1 protein is a known feedback inhibitor of IFN- $\gamma$ receptor signaling and is induced directly by viable T. gondii parasites (26). In general, the SOCS proteins, especially SOCS1, are expressed by immune cells and cells of the CNS, and have the potential to impact immune processes within the CNS, including inflammatory cytokine and chemokine production, as well as activation of microglia (33). In this study, transcripts of SOCS1 were steadily expressed during the CI stage. It is known that SOCS induces a high Arg1:iNOS activity ratio and suppresses T-cell proliferation (34). Moreover, SOCS1 contributes to the inhibition of IFN- $\gamma$ signaling and NO production without the dependency of TLR stimulation (35). In other words, due to upregulation of SOCS1 by IL-4-dependent M2 macrophage activation (34), the role of SOCS1 in the present study is sufficiently predictable for the direct increase in the Arg1:iNOS activity ratio, the decrease in transcripts of T-cell differentiation markers and Stat 1 phosphorylation, and the increase in transcripts of T-cell exhaustion markers, suggesting modulations of the host-parasite relationship between parasite control and tissue damage in the brain.

Taken together, these results highlight the critical importance of M1-type microglia for the control of T. gondii as well as the limitation of STAT1 phosphorylation and the induction of SOCS1 to reduce detrimental inflammatory immune responses. Moreover, these results provide new insights into the characteristics of infection immunity through the host-parasite relationship during CI of $T$. gondii. In other words, T. gondii infection basically induces M1-type polarization of microglial cells, the limitation of IFN- $\boldsymbol{\gamma}$-mediated inflammatory responses, including T-cell differentiation and Stat 1 phosphorylation, and

\section{REFERENCES}

1. Wohlfert EA, Blader IJ, Wilson EH. Brains and Brawn: Toxoplasma infections of the central nervous system and skeletal muscle. Trends Parasitol (2017) 33:519-31. doi:10.1016/j.pt.2017.04.001

2. Mordue DG, Monroy F, La Regina M, Dinarello CA, Sibley LD. Acute toxoplasmosis leads to lethal overproduction of Th1 cytokines. J Immunol (2001) 167:4574-84. doi:10.4049/jimmunol.167.8.4574

3. Jensen KD, Wang Y, Wojno ED, Shastri AJ, Hu K, Cornel L, et al. Toxoplasma polymorphic effectors determine macrophage polarization and intestinal inflammation. Cell Host Microbe (2011) 9:472-83. doi:10.1016/j.chom. 2011.04.015

4. Rozenfeld C, Martinez R, Seabra S, Sant'anna C, Goncalves JG, Bozza M, et al. Toxoplasma gondii prevents neuron degeneration by interferongamma-activated microglia in a mechanism involving inhibition of inducible nitric oxide synthase and transforming growth factor-betal production by infected microglia. Am J Pathol (2005) 167:1021-31. doi:10.1016/ S0002-9440(10)61191-1

5. Suzuki Y. Host resistance in the brain against Toxoplasma gondii. J Infect Dis (2002) 185(Suppl 1):S58-65. doi:10.1086/337999

6. Jankovic D, Kugler DG, Sher A. IL-10 production by CD4+ effector T cells: a mechanism for self-regulation. Mucosal Immunol (2010) 3:239-46. doi:10.1038/mi.2010.8

7. Sarciron ME, Gherardi A. Cytokines involved in Toxoplasmic encephalitis. Scand J Immunol (2000) 52:534-43. doi:10.1046/j.1365-3083.2000.00817.x

8. Jung BK, Pyo KH, Shin KY, Hwang YS, Lim H, Lee SJ, et al. Toxoplasma gondii infection in the brain inhibits neuronal degeneration and learning and memory impairments in a murine model of Alzheimer's disease. PLoS One (2012) 7:e33312. doi:10.1371/journal.pone.0033312 the reduction of iNOS/Arg1 ratio mediated by Socs1 induction. These phenomena are only expected by infection immunity due to the difference in the levels of effector molecules via the in vitro cellular response with TLA. In particular, this study revealed the characteristics of immune regulation in infection immunity by observing the persistent changes in the immune environment during a CI rather than at any one time.

\section{ETHICS STATEMENT}

This study protocol was approved by the Ethics Committee of Seoul National University and conducted in strict accordance with the Guidelines for Animal Experiments (SNUIBC-R110302-1). All surgeries were performed under anesthesia and all efforts were made to ensure minimal animal suffering.

\section{AUTHOR CONTRIBUTIONS}

$\mathrm{YH}$ and E-HS conceived and designed the experiments. J-HS and YH performed the experiments. J-HS, J-PY, and E-HS analyzed the data. B-KJ, SL, and E-HS contributed reagents/materials/ analysis tools. E-HS wrote the paper.

\section{FUNDING}

This work was supported by the Basic Science Research Program of the National Research Foundation of Korea funded by the Ministry of Education, Science and Technology (grant no. NRF-2015R1D1A1A01058902) and the Seoul National University Bundang Hospital Research Fund (grant no. 02-2012-040).

9. Orihuela R, McPherson CA, Harry GJ. Microglial M1/M2 polarization and metabolic states. Br J Pharmacol (2016) 173:649-65. doi:10.1111/ bph.13139

10. Nguyen TD, Bigaignon G, Van BroeckJ, Vercammen M, Nguyen TN, Delmee M, et al. Acute and chronic phases of Toxoplasma gondii infection in mice modulate the host immune responses. Infect Immun (1998) 66:2991-5.

11. Hester J, Mullins J, Sa Q, Payne L, Mercier C, Cesbron-Delauw MF, et al. Toxoplasma gondii antigens recognized by IgG antibodies differ between mice with and without active proliferation of tachyzoites in the brain during the chronic stage of infection. Infect Immun (2012) 80:3611-20. doi:10.1128/ IAI.00604-12

12. El Kasmi KC, Qualls JE, Pesce JT, Smith AM, Thompson RW, Henao-Tamayo M, et al. Toll-like receptor-induced arginase 1 in macrophages thwarts effective immunity against intracellular pathogens. Nat Immunol (2008) 9:1399-406. doi:10.1038/ni.1671

13. Guillemin GJ, Brew BJ. Microglia, macrophages, perivascular macrophages, and pericytes: a review of function and identification. J Leukoc Biol (2004) 75:388-97. doi:10.1189/jlb.0303114

14. Rosowski EE, Lu D, Julien L, Rodda L, Gaiser RA, Jensen KD, et al. Strainspecific activation of the NF-kappaB pathway by GRA15, a novel Toxoplasma gondii dense granule protein. J Exp Med (2011) 208:195-212. doi:10.1084/ jem.20100717

15. Harris TH, Wilson EH, Tait ED, Buckley M, Shapira S, Caamano J, et al. NF-kappaB1 contributes to T cell-mediated control of Toxoplasma gondii in the CNS. J Neuroimmunol (2010) 222:19-28. doi:10.1016/j.jneuroim. 2009.12.009

16. Rozenfeld C, Martinez R, Figueiredo RT, Bozza MT, Lima FR, Pires AL, et al. Soluble factors released by Toxoplasma gondii-infected astrocytes down-modulate nitric oxide production by gamma interferon-activated 
microglia and prevent neuronal degeneration. Infect Immun (2003) 71:2047-57. doi:10.1128/IAI.71.4.2047-2057.2003

17. Tait ED, Hunter CA. Advances in understanding immunity to Toxoplasma gondii. Mem Inst Oswaldo Cruz (2009) 104:201-10. doi:10.1590/S007402762009000200013

18. Christian DA, Koshy AA, Reuter MA, Betts MR, Boothroyd JC, Hunter CA. Use of transgenic parasites and host reporters to dissect events that promote interleukin-12 production during toxoplasmosis. Infect Immun (2014) 82:4056-67. doi:10.1128/IAI.01643-14

19. Lu F, Huang S, Kasper LH. Interleukin-10 and pathogenesis of murine ocular toxoplasmosis. Infect Immun (2003) 71:7159-63. doi:10.1128/IAI.71. 12.7159-7163.2003

20. Kamerkar S, Davis PH. Toxoplasma on the brain: understanding host-pathogen interactions in chronic CNS infection. J Parasitol Res (2012) 2012:589295. doi:10.1155/2012/589295

21. Pyo KH, Jung BK, Xin CF, Lee YW, Chai JY, Shin EH. Prominent IL-12 production and tumor reduction in athymic nude mice after Toxoplasma gondii lysate antigen treatment. Korean J Parasitol (2014) 52:605-12. doi:10.3347/ kjp.2014.52.6.605

22. Pyo KH, Lee YW, Lim SM, Shin EH. Immune adjuvant effect of a Toxoplasma gondii profilin-like protein in autologous whole-tumor-cell vaccination in mice. Oncotarget (2016) 7:74107-19. doi:10.18632/oncotarget. 12316

23. Rosowski EE, Saeij JP. Toxoplasma gondii clonal strains all inhibit STAT1 transcriptional activity but polymorphic effectors differentially modulate IFNgamma induced gene expression and STAT1 phosphorylation. PLoS One (2012) 7:e51448. doi:10.1371/journal.pone.0051448

24. Legat A, Speiser DE, Pircher H, Zehn D, Fuertes Marraco SA. Inhibitory receptor expression depends more dominantly on differentiation and activation than "exhaustion" of human CD8 T cells. Front Immunol (2013) 4:455. doi:10.3389/fimmu.2013.00455

25. Gavrilescu LC, Butcher BA, Del Rio L, Taylor GA, Denkers EY. STAT1 is essential for antimicrobial effector function but dispensable for gamma interferon production during Toxoplasma gondii infection. Infect Immun (2004) 72:1257-64. doi:10.1128/IAI.72.3.1257-1264.2004

26. Zimmermann S, Murray PJ, Heeg K, Dalpke AH. Induction of suppressor of cytokine signaling-1 by Toxoplasma gondii contributes to immune evasion in macrophages by blocking IFN-gamma signaling. J Immunol (2006) 176:1840-7. doi:10.4049/jimmunol.176.3.1840
27. Loke P, Allison JP. PD-L1 and PD-L2 are differentially regulated by Th1 and Th2 cells. Proc Natl Acad Sci U S A (2003) 100:5336-41. doi:10.1073/ pnas.0931259100

28. Bhadra R, Gigley JP, Weiss LM, Khan IA. Control of Toxoplasma reactivation by rescue of dysfunctional CD8+ T-cell response via PD-1-PDL-1 blockade. Proc Natl Acad Sci U S A (2011) 108:9196-201. doi:10.1073/pnas.1015298108

29. McBerry C, Dias A, Shryock N, Lampe K, Gutierrez FR, Boon L, et al. PD-1 modulates steady-state and infection-induced IL-10 production in vivo. Eur J Immunol (2014) 44:469-79. doi:10.1002/eji.201343658

30. Bhadra R, Gigley JP, Khan IA. PD-1-mediated attrition of polyfunctional memory CD8+ T cells in chronic toxoplasma infection. J Infect Dis (2012) 206:125-34. doi:10.1093/infdis/jis304

31. Lan X, Han X, Li Q, Yang QW, Wang J. Modulators of microglial activation and polarization after intracerebral haemorrhage. Nat Rev Neurol (2017) 13:420-33. doi:10.1038/nrneurol.2017.69

32. Denkers EY. From cells to signaling cascades: manipulation of innate immunity by Toxoplasma gondii. FEMS Immunol Med Microbiol (2003) 39:193-203. doi:10.1016/S0928-8244(03)00279-7

33. Baker BJ, Akhtar LN, Benveniste EN. SOCS1 and SOCS3 in the control of CNS immunity. Trends Immunol (2009) 30:392-400. doi:10.1016/j.it.2009.07.001

34. Whyte CS, Bishop ET, Ruckerl D, Gaspar-Pereira S, Barker RN, Allen JE, et al. Suppressor of cytokine signaling (SOCS)1 is a key determinant of differential macrophage activation and function. J Leukoc Biol (2011) 90:845-54. doi:10.1189/jlb.1110644

35. Stutz A, Kessler H, Kaschel ME, Meissner M, Dalpke AH. Cell invasion and strain dependent induction of suppressor of cytokine signaling-1 by Toxoplasma gondii. Immunobiology (2012) 217:28-36. doi:10.1016/j.imbio. 2011.08.008

Conflict of Interest Statement: The authors declare that the research was conducted in the absence of any commercial or financial relationships that could be construed as a potential conflict of interest.

Copyright (C) 2018 Hwang, Shin, Yang, Jung, Lee and Shin. This is an open-access article distributed under the terms of the Creative Commons Attribution License (CC $B Y)$. The use, distribution or reproduction in other forums is permitted, provided the original author(s) and the copyright owner are credited and that the original publication in this journal is cited, in accordance with accepted academic practice. No use, distribution or reproduction is permitted which does not comply with these terms. 